quatrième série-tome 41 fascicule 5 septembre-octobre 2008

$$
\begin{aligned}
& \text { ANNALES } \\
& \text { SCIENTIFIQUES } \\
& \text { de } \\
& \text { L'ECOLE } \\
& \text { NORMALE } \\
& \text { SUPÉRIEURE }
\end{aligned}
$$

\title{
Alberto MÍNGUEZ
}

Correspondance de Howe explicite : paires duales de type II 
Ann. Scient. Éc. Norm. Sup.

$4^{\mathrm{e}}$ série, t. 41,2008, p. 717 à 741

\title{
CORRESPONDANCE DE HOWE EXPLICITE : PAIRES DUALES DE TYPE II*
}

\author{
PAR Alberto mínguez
}

RÉSUMÉ. - Dans cet article, nous proposons une nouvelle méthode pour démontrer la bijectivité de la correspondance de Howe pour les paires duales du type $\left(\mathrm{GL}_{n}, \mathrm{GL}_{m}\right)$ sur un corps $F$ localement compact non archimédien. La preuve est basée sur une étude soigneuse de la filtration de Kudla [11] ainsi que sur les résultats de [13] à propos de l'irréductibilité d'une représentation induite parabolique. Elle est valable pour $F$ de caractéristique quelconque et nous permet d'expliciter la bijection en termes des paramètres de Langlands. Elle généralise donc les résultats de [20] et répond totalement aux questions étudiées dans [15] et [16] pour les paires duales de type II.

Abstract. - In this article, we give a new method for proving Howe correspondence in the case of dual pairs of type $\left(\mathrm{GL}_{n}, \mathrm{GL}_{m}\right)$ over a non-Archimedean locally compact field $F$. The proof consists in combining a study on Kudla's filtration [11] with the results of [13] about the irreducibility of a parabolically induced representation. The proof is valid for $F$ of any characteristic and allows us to make the correspondence explicit in terms of Langlands parameters. Hence it generalizes the results of [20] and answers completely all questions studied in [15] and [16] for dual pairs of type II.

\section{Introduction}

Soit $F$ un corps commutatif localement compact non archimédien de caractéristique résiduelle $p>0$. Soit $\psi: F \rightarrow \mathbb{C}^{\times}$un caractère additif non trivial de $F$. Si $W$ est un espace vectoriel symplectique sur $F$, de dimension finie, on dispose du groupe métaplectique $\widetilde{S p}(W)$, qui est un revêtement à deux feuillets du groupe symplectique $S p(W)$, et d'une représentation $(\omega, S)$ de $\widetilde{S p}(W)$ canoniquement attachée à $\psi$, dite représentation de Weil ou métaplectique, sur un espace de fonctions $S$ à valeurs complexes. Soit $\left(G, G^{\prime}\right)$ une paire duale réductive ( $c f$. [14, 1.I.17]) dans $S p(W)$ : ou bien $\left(G, G^{\prime}\right)$ est une paire de groupes classiques -symplectique,

*Partially supported by MTM2004-07203-C02-01 and FEDER. 
orthogonal, unitaire- (paires duales de type I) ou bien une paire de groupes linéaires (paires duales de type II). Notons $\widetilde{G}$ et $\widetilde{G^{\prime}}$ leurs images réciproques dans $\widetilde{S p}(W)$.

Soit $\pi$ une représentation lisse irréductible de $\widetilde{G}$ quotient de $\omega$. Notons $S[\pi]$ le plus grand quotient $\pi$-isotypique de $\omega$. Il est de la forme

$$
S[\pi]=\pi_{1} \otimes \Theta(\pi),
$$

en tant que $\widetilde{G} \times \widetilde{G^{\prime}}$-module, où $\Theta(\pi)$ est une représentation lisse de longueur finie de $\widetilde{G^{\prime}}$.

Roger Howe et Jean-Loup Waldspurger [19], [14] ont prouvé que, dans le cas où $p$ est impair et où $\left(G, G^{\prime}\right)$ est de type $\mathrm{I}$, si $\Theta(\pi) \neq 0$, alors $\Theta(\pi)$ a un unique quotient irréductible, noté $\theta(\pi)$. L'application $\pi \mapsto \theta(\pi)$ est une bijection entre l'ensemble des représentations lisses irréductibles $\pi$ de $\widetilde{G}$ telles que $\Theta(\pi) \neq 0$ et l'ensemble des représentations lisses irréductibles $\pi^{\prime}$ de $\widetilde{G^{\prime}}$ telles que $\Theta\left(\pi^{\prime}\right) \neq 0$. Elle est appelée la correspondance de Howe. Nous nous proposons de montrer un théorème similaire pour les paires duales de type II, valable pour tout $p$, et d'expliciter, en termes des paramètres de Langlands, la correspondance $\pi \mapsto \theta(\pi)$, ce qui détermine l'ensemble des représentations $\pi$ telles que $\Theta(\pi) \neq 0$.

Dans le cas des paires duales de type II, Roger Howe, dans un manuscrit non publié, avait prouvé la bijectivité de la correspondance. Notre méthode, différente, rend, de plus, la correspondance explicite.

Passons à une présentation plus détaillée des résultats :

Soit $D$ une algèbre à division de centre $F$ de dimension finie $d^{2}$ sur $F$ et soient $n$ et $m$ des entiers strictement positifs. On note $\mathcal{M}_{n, m}\left(\right.$ resp. $\mathcal{M}_{n}$ ) l'ensemble des matrices $n \times m$ (resp. $n \times n)$ à coefficients dans $D$. Le groupe $\mathrm{GL}_{n}(D)$ des matrices inversibles dans $\mathcal{M}_{n}$ sera noté $G_{n}$. Notons $\omega_{n, m}$ la restriction de la représentation métaplectique à la paire duale $G_{n} \times G_{m}$ (voir (1.4) pour plus de détails).

Le résultat principal de cet article est le théorème suivant.

THÉORÈME 1 (voir corollaire 6.3). - Soit $\pi$ une représentation lisse irréductible de $G_{n}$.

1. Si $\operatorname{Hom}_{G_{n}}\left(\omega_{n, m}, \pi\right) \neq 0$, alors il existe une unique représentation lisse irréductible $\pi^{\prime}$ de $G_{m}$ telle que

$$
\operatorname{Hom}_{G_{n} \times G_{m}}\left(\omega_{n, m}, \pi \otimes \pi^{\prime}\right) \neq 0 .
$$

De plus, $\operatorname{dim}\left(\operatorname{Hom}_{G_{n} \times G_{m}}\left(\omega_{n, m}, \pi \otimes \pi^{\prime}\right)\right)=1$.

2. Supposons $n \leq m$. Alors $\operatorname{Hom}_{G_{n}}\left(\omega_{n, m}, \pi\right) \neq 0$ et, si $\pi$ est le quotient de Langlands ( $c f$. section 6) de l'induite parabolique $\tau_{1} \times \cdots \times \tau_{N}$, où $\tau_{1}, \ldots, \tau_{N}$ sont des représentations essentiellement de carré intégrable, alors $\pi^{\prime}$ est le quotient de Langlands de

$$
\nu^{-\frac{m-n-1}{2}} \times \cdots \times \nu^{\frac{m-n-1}{2}} \times \widetilde{\tau_{1}} \times \cdots \times \widetilde{\tau_{N}},
$$

où, pour toute représentation $\tau, \widetilde{\tau}$ désigne sa contragrédiente.

Dans le cas particulier où $D=F$, notons $\pi^{*}$ les paramètres galoisiens de Langlands de la représentation $\pi$, c'est-à-dire, $\pi^{*}$ est la représentation de degré $n$ du groupe de Weil $W_{F}$ d'une clôture algébrique $\bar{F}$ sur $F$ qui correspond par [8] ou [9] à $\pi$. Alors les paramètres de $\theta(\pi)$ sont $\widetilde{\pi}^{*} \oplus 1_{m-n}^{*}$ où on a noté $1_{m-n}^{*}$ les paramètres galoisiens de la représentation triviale de $G_{m-n}$.

La preuve du théorème 1 se décompose en trois parties. D'abord, la théorie des fonctions zêta de Godement-Jacquet [6] nous fournit un entrelacement entre $\omega_{n, n}$ et $\pi \otimes \tilde{\pi}$ pour toute 
représentation lisse irréductible $\pi$ de $G_{n}$, ce qui implique, avec un argument classique ( $c f$. [14, 3.III.5]), que, si $n \leq m$, alors $\operatorname{Hom}_{G_{n}}\left(\omega_{n, m}, \pi\right) \neq 0$.

Pour montrer l'unicité de la représentation $\theta(\pi)$, on a besoin d'utiliser l'article [13] où il est prouvé que l'induite parabolique d'une représentation irréductible a, dans beaucoup de cas, une seule sous-représentation irréductible.

Dans la section 2, on décrit explicitement le bord de la représentation métaplectique : le concept de bord apparaît dans [7, Definition 4.6] pour les paires duales de type I. On calcule une filtration naturelle de la représentation métaplectique et on dit qu'une représentation n'apparaît pas dans le bord si elle provient du dernier cran. On trouve que, pour toute telle représentation $\pi$, la représentation $\theta(\pi)$ est unique.

Après, dans la section 3, on s'inspire de l'article [11], et on calcule une filtration des foncteurs de Jacquet de la représentation métaplectique. Ceci nous permet de montrer dans les sections 4 et 5 , par récurrence, l'unicité de la représentation $\theta(\pi)$, pour les bonnes représentations $\pi$. Les mauvaises représentations sont celles qui ont un foncteur de Jacquet bien précis. Or, ces représentations n'apparaissent pas dans le bord de la représentation métaplectique!

Pour montrer le paramétrage de la correspondance on a, à nouveau, deux cas. Par récurrence, le cas des bonnes représentations n'est pas très difficile et découle de [13, Corollaire A.3]. Pour les autres, on utilise, dans la section 9, des propriétés subtiles de la classification de Zelevinsky-Tadić des représentations irréductibles en termes de segments.

Je voudrais particulièrement remercier Colette Mœglin qui m'a prodigué nombre de conseils et idées, ainsi que Guy Henniart pour toutes ses suggestions et critiques. Je remercie aussi Takuya Konno, Goran Muić et Vincent Sécherre pour les remarques et les corrections qu'ils m'ont faites à propos de cet article.

\section{Préliminaires}

Soient $F$ un corps commutatif localement compact non archimédien de caractéristique résiduelle $p>0, D$ une algèbre à division de centre $F$ et de dimension finie $d^{2}$ sur $F$.

Soient $n, m$ deux entiers strictement positifs. On note $\mathcal{M}_{n, m}$ (resp. $\mathcal{M}_{n}$ ) l'ensemble des matrices $n \times m$ (resp. $n \times n$ ) à coefficients dans $D$ et $\operatorname{Nrd}: \mathcal{M}_{n} \rightarrow F$ la norme réduite. Le groupe $\mathrm{GL}_{n}(D)$ des matrices inversibles dans $\mathcal{M}_{n}$ sera noté $G_{n}$. Le groupe trivial sera noté $G_{0}$.

À toute partition (ordonnée) $\alpha=\left(n_{1}, \ldots, n_{r}\right)$ de l'entier $n$, correspond une décomposition en blocs des matrices carrées d'ordre $n$. On notera $M_{\alpha}$ le sous-groupe de $G_{n}$ formé des matrices inversibles diagonales par blocs (les blocs étant dans $\mathcal{M}_{n_{i}, n_{i}}$, pour $1 \leq i \leq r$ ), $P_{\alpha}$ (resp. $\bar{P}_{\alpha}$ ) le sous-groupe formé des matrices triangulaires supérieures (resp. inférieures) par blocs, et $U_{\alpha}$ le sous-groupe de $P_{\alpha}$ formé des éléments dont les blocs diagonaux sont des matrices unité. Le sous-groupe $\bar{P}_{\alpha}$ est conjugué à $P_{\bar{\alpha}}$ dans $G_{n}$ avec $\bar{\alpha}=\left(n_{r}, \ldots, n_{1}\right)$.

Dans cet article, on ne considérera que des représentations lisses complexes et le mot représentation voudra toujours dire représentation lisse complexe. On notera $\operatorname{Irr}\left(G_{n}\right)$ l'ensemble des classes d'équivalence des représentations irréductibles de $G_{n}$. La représentation triviale de $G_{n}$ sera notée $1_{n}$.

Si $\pi$ et $\pi^{\prime}$ sont deux représentations d'un groupe $G$, on notera

$$
\operatorname{Hom}_{G}\left(\pi, \pi^{\prime}\right)
$$


l'espace des entrelacements entre $\pi$ et $\pi^{\prime}$. On omettra l'indice $G$ quand il n'y a pas de confusion.

On note $\sharp-r_{n_{1}, \ldots, n_{r}}^{G_{n}}$ (resp. $\left.\sharp-\bar{r}_{n_{1}, \ldots, n_{r}}^{G_{n}}\right)$ le foncteur de Jacquet non normalisé associé au parabolique standard $P_{\alpha}$ (resp. $\left.\bar{P}_{\alpha}\right)$. On note

$$
\begin{aligned}
r_{n_{1}, \ldots, n_{r}}^{G_{n}} & =\delta_{P_{\alpha}}^{-1 / 2} \sharp-r_{n_{1}, \ldots, n_{r}}^{G_{n}}, \\
\text { (resp. } \bar{r}_{n_{1}, \ldots, n_{r}}^{G_{n}} & \left.=\delta_{\bar{P}_{\alpha}}^{-1 / 2} \sharp-\bar{r}_{n_{1}, \ldots, n_{r}}^{G_{n}}\right),
\end{aligned}
$$

le foncteur de Jacquet normalisé.

Etant donnée une représentation $\rho_{i}$ de chaque $G_{n_{i}}$, on notera

$$
\sharp-\operatorname{ind}_{P_{\alpha}}^{G_{n}}\left(\rho_{1} \otimes \cdots \otimes \rho_{r}\right)
$$

l'induite parabolique non normalisée, où on a prolongé la représentation $\rho_{1} \otimes \cdots \otimes \rho_{r}$ trivialement $\operatorname{sur} U_{\alpha}$.

On note aussi $\rho_{1} \times \cdots \times \rho_{r}$ la représentation

$$
\operatorname{ind}_{P_{\alpha}}^{G_{n}}\left(\rho_{1} \otimes \cdots \otimes \rho_{r}\right)=\delta_{P_{\alpha}}^{1 / 2} \sharp-\operatorname{ind}_{P_{\alpha}}^{G_{n}}\left(\rho_{1} \otimes \cdots \otimes \rho_{r}\right),
$$

induite parabolique normalisée.

Soit $\pi$ une représentation de $G_{n}$; on a un isomorphisme canonique (réciprocité de Frobenius) :

$$
\operatorname{Hom}\left(\pi, \rho_{1} \times \cdots \times \rho_{r}\right) \simeq \operatorname{Hom}\left(r_{n_{1}, \ldots, n_{r}}^{G_{n}}(\pi), \rho_{1} \otimes \cdots \otimes \rho_{r}\right) .
$$

On a une formule similaire pour l'induction non normalisée et le foncteur de Jacquet non normalisé. On dispose aussi d'un isomorphisme de réciprocité à la Casselman ( $c f$. [1, Theorem 20] ou bien [4]) :

$$
\operatorname{Hom}\left(\rho_{1} \times \cdots \times \rho_{r}, \pi\right) \simeq \operatorname{Hom}\left(\rho_{1} \otimes \cdots \otimes \rho_{r}, \bar{r}_{n_{1}, \ldots, n_{r}}^{G_{n}}(\pi)\right) .
$$

Pour l'induction parabolique et le foncteur de Jacquet non normalisés, la formule précédente devient :

$$
\begin{aligned}
\operatorname{Hom}\left(\sharp-\operatorname{ind}_{P_{\alpha}}^{G_{n}}\left(\rho_{1} \otimes \cdots \otimes \rho_{r}\right), \pi\right) & \simeq \\
& \operatorname{Hom}\left(\rho_{1} \otimes \cdots \otimes \rho_{r}, \delta_{P_{\alpha}} \sharp-\bar{r}_{n_{1}, \ldots, n_{r}}^{G_{n}}(\pi)\right) .
\end{aligned}
$$

Soient $n, t \in \mathbb{Z}, 1 \leq t \leq n, \pi \in \operatorname{Irr}\left(G_{n}\right), \chi \in \operatorname{Irr}\left(G_{t}\right)$. On notera $\operatorname{Jac}_{\chi}(\pi) \neq 0$ (resp. $\left.\overline{\operatorname{Jac}}_{\chi}(\pi) \neq 0\right)$ s'il existe $\rho \in \operatorname{Irr}\left(G_{n-t}\right)$ tel que $\operatorname{Hom}(\pi, \chi \times \rho) \neq 0(\operatorname{resp} . \operatorname{Hom}(\pi, \rho \times \chi) \neq 0)$.

On utilisera à plusieurs reprises la proposition suivante [13, Proposition 7.1] :

Proposition 1.1. - Soient $n, m$ deux entiers positifs, $n \leq m, \pi \in \operatorname{Irr}\left(G_{n}\right), \pi^{\prime} \in \operatorname{Irr}\left(G_{m}\right)$ et $\rho$ une représentation cuspidale irréductible de $G_{m-n}$. Les conditions suivantes sont équivalentes:

1. $\operatorname{Hom}\left(\pi^{\prime}, \pi \times \rho\right) \neq 0$;

2. $\operatorname{Hom}\left(\rho \times \pi, \pi^{\prime}\right) \neq 0$.

COROLlaire 1.2. - Soient $\pi, \pi^{\prime}$ deux représentations irréductibles et $\rho$ une représentation cuspidale. Les conditions suivantes sont équivalentes :

1. $\operatorname{Hom}\left(\pi^{\prime}, \pi \times \rho \times \cdots \times \rho\right) \neq 0$;

2. $\operatorname{Hom}\left(\rho \times \cdots \times \rho \times \pi, \pi^{\prime}\right) \neq 0$. 
Démonstration. - Puisque, par [13, Théorème 5.1], $\pi \times \rho \times \cdots \times \rho$ n'a qu'un seul sousmodule irréductible et que, par [13, Théorème 5.6], $\rho \times \cdots \times \rho \times \pi$ n'a qu'un seul quotient irréductible, le corollaire découle de la proposition précédente par récurrence.

Si $X$ est un espace localement profini, on note $S(X)$ l'espace vectoriel des fonctions $\Phi: X \rightarrow \mathbb{C}$ localement constantes à support compact. Le lemme suivant sera utilisé dans le calcul explicite de la correspondance :

Lemme 1.3. - Soit $X$ un espace localement profini, $X^{\prime}$ un sous-espace fermé de $X$. Supposons qu'un groupe localement profini $G$ agisse de façon continue sur $X$ et que $G \cdot X^{\prime}=X$. Notons $H$ le stabilisateur de $X^{\prime}$ dans $G$. Notons aussi $\pi$ la représentation naturelle (cf. [2, §1.2.2]) de $G$ dans $S(X)$ et $\rho$ la représentation naturelle de $H$ dans $S\left(X^{\prime}\right)$. Alors :

$$
\pi \simeq \sharp-\operatorname{ind}_{H}^{G}(\rho) .
$$

Démonstration. - Posons

$$
\begin{aligned}
\Xi: \pi & \rightarrow \sharp-\operatorname{ind}_{H}^{G}(\rho) \\
\phi & \mapsto\left(\left.g \mapsto(\pi(g) \phi)\right|_{X^{\prime}}\right) .
\end{aligned}
$$

$\Xi$ est bien défini car $X^{\prime}$ est fermé dans $X(c f .[2, \S 1.1 .8])$ et c'est un entrelacement entre $\pi$ et $\sharp-\operatorname{ind}_{H}^{G}(\rho)$.

Construisons une inverse : Soit $f \in \sharp-\operatorname{ind}_{H}^{G}(\rho)$. On définit $\phi_{f} \in S(X)$ par

$$
\phi_{f}(x)=f(g)\left(x^{\prime}\right),
$$

si $x=g \cdot x^{\prime}$ et $g \in G$ et $x^{\prime} \in X^{\prime}$. Puisque $G \cdot X^{\prime}=X$, de tels couples $\left(g, x^{\prime}\right)$ existent et, si $g_{1}, g_{2} \in G$ et $x_{1}^{\prime}, x_{2}^{\prime} \in X^{\prime}$ sont tels que $x=g_{1} \cdot x_{1}^{\prime}=g_{2} \cdot x_{2}^{\prime}$, alors $x_{1}^{\prime}=g_{1}^{-1} g_{2} \cdot x_{2}^{\prime}$ et donc, si l'on pose $h=g_{1}^{-1} g_{2}$, on a que $h \in H$.

Ainsi

$$
\begin{aligned}
f\left(g_{1}\right)\left(x_{1}^{\prime}\right) & =f\left(g_{1}\right)\left(g_{1}^{-1} g_{2} \cdot x_{2}^{\prime}\right) \\
& =\rho(h) f\left(g_{1}\right)\left(x_{2}^{\prime}\right) \\
& =f\left(g_{1} h\right)\left(x_{2}^{\prime}\right) \\
& =f\left(g_{2}\right)\left(x_{2}^{\prime}\right) .
\end{aligned}
$$

Donc $\phi_{f}$ est bien définie et le morphisme $f \mapsto \phi_{f}$ est un entrelacement entre $\sharp-\operatorname{ind}_{H}^{G}(\rho)$ et $\pi$, inverse de $\Xi$.

On note $S_{n, m}=S\left(\mathcal{M}_{n, m}\right)$ l'espace vectoriel des fonctions $\Phi$ de $\mathcal{M}_{n, m}$ dans $\mathbb{C}$, localement constantes à support compact. On utilisera le modèle suivant de la représentation métaplectique $\omega_{n, m}$ restreinte à la paire duale $G_{n} \times G_{m}(c f \text {. [14, 2.II.6] })^{(1)}$ :

$$
\omega_{n, m}\left(g, g^{\prime}\right)=\nu(g)^{\frac{-m}{2}} \sigma_{n, m}\left(g, g^{\prime}\right) \nu\left(g^{\prime}\right)^{\frac{n}{2}}
$$

(1) Dans [14, 3.III.1], on utilise une définition légèrement différente :

$$
M\left(g, g^{\prime}\right) \Phi(x)=\nu(g)^{\frac{m}{2}} \Phi\left({ }^{t} g x g^{\prime}\right) \nu\left(g^{\prime}\right)^{\frac{n}{2}} .
$$

Cette définition peut induire à confusion et, à notre avis, n'est pas la plus naturelle. Dans le cas où $D$ n'est pas commutatif, si $g \in G_{n}$ alors ${ }^{t} g \in G_{n}^{0}, G_{n}^{0}$ étant le groupe opposé, et il peut sembler, par exemple dans le cas où $n=1$, qu'on ne définit pas bien une action à gauche. Si $D=F, M\left(g, g^{\prime}\right)=\omega_{n, m}\left({ }^{t} g^{-1}, g^{\prime}\right)$ et $\pi \otimes \pi^{\prime}$ est un quotient de $M$ si, et seulement si, $\widetilde{\pi} \otimes \pi^{\prime}$ est un quotient de $\omega_{n, m}$ 
où on note $\nu=|\operatorname{Nrd}|_{F}$, la valeur absolue de la norme réduite et

$$
\sigma_{n, m}: G_{n} \times G_{m} \rightarrow \operatorname{GL}\left(S_{n, m}\right)
$$

la représentation naturelle de $G_{n} \times G_{m}$ définie par

$$
\sigma_{n, m}\left(g, g^{\prime}\right) \Phi(x)=\Phi\left(g^{-1} x g^{\prime}\right),
$$

pour $g \in G_{n}, g^{\prime} \in G_{m}, x \in \mathcal{M}_{n, m}, \Phi \in S_{n, m}$.

Il est plus naturel de travailler avec la représentation métaplectique tordue $\sigma_{n, m}$ et de calculer ses quotients irréductibles. On déduira ensuite immédiatement les résultats pour la représentation $\omega_{n, m}$.

Il est aussi très pratique d'utiliser la notation suivante : on a deux groupes linéaires agissant, par multiplication, sur un espace de matrices à gauche et à droite. Dorénavant, pour différencier ces deux actions, on notera $G^{\prime}, P^{\prime}$ et $U^{\prime}$ les groupes linéaire, parabolique et unipotent respectivement, agissant à droite et on gardera les notations $G, P$ et $U$ pour ces groupes quand ils agissent à gauche. De même, en cas d'ambiguïté, on notera $\nu^{\prime}$ le caractère $\nu$ quand il agit sur $G^{\prime}$. Cela peut sembler une notation un peu artificielle mais elle facilite énormément la compréhension des calculs.

On permet les cas $m=0$ ou $n=0$ (avec $G_{0}=0$ ou $G_{0}^{\prime}=0$ ) pour lesquels $\mathcal{M}_{n, 0}=0$, $\mathcal{M}_{0, m}=0$ et $\sigma_{n, 0}$ est la représentation triviale de $G_{n}$ et $\sigma_{0, m}$ est la représentation triviale de $G_{m}^{\prime}$.

\section{Le bord de la représentation métaplectique}

Dans cette section, on rappelle les résultats de [14, 3.III] et on en déduit quelques premières conséquences. On fixe des entiers positifs $n$ et $m$.

Commençons par rappeler que la théorie des fonctions zêta de Godement-Jacquet [6] nous fournit, pour toute représentation irréductible $\pi$ de $G_{n}$, un entrelacement (cf. [14, 3.III.7]) entre $\sigma_{n, n}$ et $\pi \otimes \widetilde{\pi}$. Par [14, 3.III.5], on déduit que, pour toute représentation irréductible $\pi$ de $G_{n}$, il existe un sous-quotient irréductible $\pi^{\prime}$ de $\sharp-\operatorname{ind}_{P_{m-n, n}^{\prime}}^{G_{m}^{\prime}}\left(1_{m-n} \otimes \widetilde{\pi}\right)$ tel que

$$
\operatorname{Hom}_{G_{n} \times G_{m}^{\prime}}\left(\sigma_{n, m}, \pi \otimes \pi^{\prime}\right) \neq 0 .
$$

Ainsi, si $n \leq m$, alors $\Theta(\pi) \neq 0$. Le problème est de montrer que ce sous-quotient $\pi^{\prime}$ est l'unique satisfaisant à (2.1) et de déterminer ses paramètres.

D'un autre côté, la représentation $\sigma_{n, m}$ admet une filtration $G_{n} \times G_{m}$-équivariante

$$
0=S_{t+1} \subset S_{t} \subset \cdots \subset S_{1} \subset S_{0}=S_{n, m},
$$

où $S_{k}$ est le sous-espace vectoriel de $S_{n, m}$ formé des fonctions dont le support est formé des matrices de rang ${ }^{(2)}$ plus grand ou égal à $k, 0 \leq k \leq t=\min (n, m)$. L'espace $S_{k+1}$ est ouvert dans $S_{k}$ par $[2, \S 1.1 .8]$ et, en appliquant le lemme 1.3 avec $X^{\prime}=\left(\begin{array}{ll}0 & 0 \\ 0 & 1_{i}\end{array}\right)$, on montre comme dans [14, 3.III.2] (en changeant le sous-groupe parabolique $P_{m-i}$ par son opposé et les transposées des matrices par les inverses des matrices) qu'on a un isomorphisme

$$
\sigma_{k}=S_{k} / S_{k+1} \simeq \sharp-\operatorname{ind}_{\bar{P}_{n-k, k} P_{m-k, k}^{\prime}}^{G_{n} G_{m}^{\prime}}\left(\mu_{k}\right),
$$

(2) On utilise la définition de rang sur une algèbre à division de [3, §10.12]

$4^{\text {e }}$ SÉRIE - TOME $41-2008-\mathrm{N}^{\mathrm{o}} 5$ 
où $\mu_{k}$ est la représentation de $\bar{P}_{n-k, k} P_{m-k, k}^{\prime}$ sur $S\left(G_{k}\right)$ définie par :

$$
\mu_{k}\left(p, p^{\prime}\right) \Phi(h)=\Phi\left(p_{4}^{-1} h p_{4}^{\prime}\right)=\rho_{k}\left(p_{4}, p_{4}^{\prime}\right) \Phi(h),
$$

pour $\Phi \in S\left(G_{k}\right), h \in G_{k}, p=\left(\begin{array}{cc}p_{1} & 0 \\ p_{3} & p_{4}\end{array}\right), p^{\prime}=\left(\begin{array}{cc}p_{1}^{\prime} & p_{2}^{\prime} \\ 0 & p_{4}^{\prime}\end{array}\right)$ et $\rho_{k}$ la représentation naturelle de $G_{k} \times G_{k}^{\prime} \operatorname{sur} S\left(G_{k}\right)$ définie par

$$
\rho_{k}\left(p_{4}, p_{4}^{\prime}\right) \Phi(h)=\Phi\left(p_{4}^{-1} h p_{4}^{\prime}\right) .
$$

Définition 2.1. - On dit que $\pi \in \operatorname{Irr}\left(G_{n}\right)$ apparaît dans le bord de la représentation $\sigma_{n, m}$ s'il existe $k<n$ tel que $\operatorname{Hom}_{G_{n}}\left(\sigma_{k}, \pi\right) \neq 0$.

Lemme 2.2. - Soient $\pi \in \operatorname{Irr}\left(G_{n}\right), \pi^{\prime} \in \operatorname{Irr}\left(G_{m}^{\prime}\right)$ telles que $\operatorname{Hom}\left(\sigma_{k}, \pi \otimes \pi^{\prime}\right) \neq 0$. Alors il existe $\tau, \tau^{\prime} \in \operatorname{Irr}\left(G_{k}\right)$ telles que

$$
\operatorname{Hom}\left(\sharp-\operatorname{ind} \bar{P}_{n-k k}^{G_{n}}\left(1_{n-k} \otimes \tau\right) \otimes \sharp-\operatorname{ind}_{P_{m-k, k}^{\prime}}^{G_{m}^{\prime}}\left(1_{m-k} \otimes \tau^{\prime}\right), \pi \otimes \pi^{\prime}\right) \neq 0 .
$$

Démonstration. - Soient $\pi \in \operatorname{Irr}\left(G_{n}\right), \pi^{\prime} \in \operatorname{Irr}\left(G_{m}^{\prime}\right)$ telles que $\operatorname{Hom}\left(\sigma_{k}, \pi \otimes \pi^{\prime}\right) \neq 0$. On a donc un entrelacement non nul de $\sharp-\operatorname{ind}{\frac{G_{n}}{P_{n-k, k}} G_{m-k, k}^{\prime}}_{m}^{\prime}\left(\mu_{k}\right)$ dans $\pi \otimes \pi^{\prime}$. Ceci équivaut, par (1.3), à l'existence d'un entrelacement non nul entre $\mu_{k}$ et $\delta_{P_{n-k, k}} \sharp-r_{n-k, k}^{G_{n}}(\pi) \otimes$ $\delta_{P_{m-k, k}^{\prime}} \sharp-\bar{r}_{m-k, k}^{G_{m}^{\prime}}\left(\pi^{\prime}\right)$.

Soit $V$ l'image d'un tel entrelacement; il existe une sous-représentation irréductible $V^{\prime}$ de $V$ et toute telle sous-représentation est de la forme $\left(1_{n-k} \otimes \tau\right) \otimes\left(1_{m-k} \otimes \tau^{\prime}\right)$ comme représentation de $\bar{P}_{n-k, k} P_{m-k, k}^{\prime}$ où $\tau$ et $\tau^{\prime}$ sont irréductibles. Par (1.3), à nouveau, on trouve le résultat.

Corollaire 2.3. - Soit $\pi \in \operatorname{Irr}\left(G_{n}\right)$. Les conditions suivantes sont équivalentes:

1. La représentation $\pi$ n'apparaît pas dans le bord de $\sigma_{n, m}$.

2. Il n'existe pas $\tau \in \operatorname{Irr}\left(G_{k}\right), k<n$, telle que

$$
\operatorname{Hom}_{G_{n}}\left(\sharp-\operatorname{ind} \bar{P}_{n-k, k}^{G_{n}}\left(1_{n-k} \otimes \tau\right), \pi\right) \neq 0 .
$$

Démonstration. - Pour l'implication directe, supposons qu'il existe $\tau \in \operatorname{Irr}\left(G_{k}\right)$, $k<n$, et un entrelacement non nul de $\left.\sharp-\operatorname{ind}{\frac{G}{P_{n-k, k}}}_{n-k} \otimes \tau\right)$ dans $\pi$. Par exactitude du foncteur $\sharp-$ ind et [14, Lemme 3.II.3], on a un morphisme surjectif de $\sigma_{k}$ dans $\sharp-\operatorname{ind} \bar{P}_{n-k, k}^{G_{n}}\left(1_{n-k} \otimes \tau\right)$ qui composé avec l'entrelacement précédent nous montre que $\operatorname{Hom}_{G_{n}}\left(\sigma_{k}, \pi\right) \neq 0$, i.e que la représentation $\pi$ apparaît dans le bord de $\sigma_{n, m}$.

Rappelons que, d'après [13, Théorème 5.1], pour toute représentation irréductible $\pi \in$ $\operatorname{Irr}\left(G_{n}\right)$, la représentation $\sharp-\operatorname{ind}_{P_{m-n, n}^{\prime}}^{G_{m}^{\prime}}\left(1_{m-n} \otimes \pi\right)$ a un unique quotient irréductible et qu'il apparaît avec multiplicité 1 dans l'induite. Le théorème suivant résume tout ce que l'on peut conclure à partir de cette filtration par le rang. 
THÉORÈme 2.4. - Soient $n, m$ des entiers positifs et supposons $n \leq m$. Soit $\pi \in \operatorname{Irr}\left(G_{n}\right)$ qui n'apparaisse pas dans le bord de $\sigma_{n, m}$. Il existe une unique représentation $\pi^{\prime} \in \operatorname{Irr}\left(G_{m}^{\prime}\right)$ telle que

$$
\operatorname{Hom}_{G_{n} \times G_{m}^{\prime}}\left(\sigma_{n, m}, \pi \otimes \pi^{\prime}\right) \neq 0 .
$$

C'est l'unique quotient irréductible de $\sharp-\operatorname{ind}_{P_{m-n, n}^{\prime}}^{G_{m}^{\prime}}\left(1_{m-n} \otimes \widetilde{\pi}\right)$. De plus,

$$
\operatorname{dim}\left(\operatorname{Hom}_{G_{n} \times G_{m}^{\prime}}\left(\sigma_{n, m}, \pi \otimes \pi^{\prime}\right)\right)=1 .
$$

Démonstration. - On avait vu au début de la section qu'il existe $\pi^{\prime} \in \operatorname{Irr}\left(G_{m}^{\prime}\right)$ telle que $\operatorname{Hom}_{G_{n} \times G_{m}^{\prime}}\left(\sigma_{n, m}, \pi \otimes \pi^{\prime}\right) \neq 0$. Montrons qu'elle est unique.

Par composition avec les morphismes $S_{j} \rightarrow \sigma_{n, m}, j=0, \ldots, n$, on obtient des morphismes $S_{j} \rightarrow \pi \otimes \pi^{\prime}$.

Soit $k$ le plus grand $j$ tel que $S_{j} \rightarrow \pi \otimes \pi^{\prime}$ ne soit pas le morphisme nul. On a donc que $\pi \otimes \pi^{\prime}$ est un quotient de $\sigma_{k}$. Par hypothèse on a $k=n$ et donc,

$$
\operatorname{Hom}\left(\sigma_{n}, \pi \otimes \pi^{\prime}\right) \neq 0 .
$$

Par définition de $\sigma_{n}$ on a alors

$$
\operatorname{Hom}\left(\sharp-\operatorname{ind}_{G_{n} \times P_{m-n, n}^{\prime}}^{G_{n} \times G_{m}^{\prime}}\left(\mu_{n}\right), \pi \otimes \pi^{\prime}\right) \neq 0 .
$$

Par (1.3) et la définition de $\mu_{n}$, on déduit que

$$
\operatorname{Hom}\left(1_{m-n} \otimes \rho_{n}, \pi \otimes \delta_{P_{m-n, n}} \sharp-\bar{r}_{m-n, n}^{G_{m}^{\prime}}\left(\pi^{\prime}\right)\right) \neq 0,
$$

d'où, par [14, Lemme 3.II.3],

$$
\operatorname{Hom}\left(1_{m-n} \otimes \tilde{\pi}, \delta_{P_{m-n, n}} \sharp-\bar{r}_{m-n, n}^{G_{m}^{\prime}}\left(\pi^{\prime}\right)\right) \neq 0,
$$

et, à nouveau par (1.3), on déduit que

$$
\operatorname{Hom}\left(\sharp-\operatorname{ind}_{P_{m-n, n}^{\prime}}^{G_{m}^{\prime}}\left(1_{m-n} \otimes \widetilde{\pi}\right), \pi^{\prime}\right) \neq 0
$$

c'est-à-dire, $\pi^{\prime}$ est l'unique quotient irréductible de $\sharp-\operatorname{ind}_{P_{m-n, n}^{\prime}}^{G_{m}^{\prime}}\left(1_{m-n} \otimes \widetilde{\pi}\right)$.

Montrons finalement que

$$
\operatorname{dim}\left(\operatorname{Hom}_{G_{n} \times G_{m}^{\prime}}\left(\sigma_{n, m}, \pi \otimes \pi^{\prime}\right)\right)=1 .
$$

Soit $\lambda \in \operatorname{Hom}_{G_{n} \times G_{m}^{\prime}}\left(\sigma_{n, m}, \pi \otimes \pi^{\prime}\right)$. La composée de $\lambda$ avec l'inclusion $\sigma_{n} \hookrightarrow \sigma_{n, m}$ n'est pas nulle. Or, par le lemme [14, Lemme 3.II.3], $\operatorname{dim}\left(\operatorname{Hom}_{G_{n} \times G_{m}^{\prime}}\left(\sigma_{n}, \pi \otimes \pi^{\prime}\right)\right)=1$, et donc cette composée est unique à homothéthie près. Ainsi, si

$$
\operatorname{dim}\left(\operatorname{Hom}_{G_{n} \times G_{m}^{\prime}}\left(\sigma_{n, m}, \pi \otimes \pi^{\prime}\right)\right)>1,
$$

on pourrait construire, par combinaison linéaire, un morphisme non nul

$$
\lambda \in \operatorname{Hom}_{G_{n} \times G_{m}^{\prime}}\left(\sigma_{n, m}, \pi \otimes \pi^{\prime}\right)
$$

tel que sa composée avec l'inclusion $\sigma_{n} \hookrightarrow \sigma_{n, m}$ soit nulle. Il existe alors $k<n$ tel que $\operatorname{Hom}_{G_{n}}\left(\sigma_{k}, \pi\right) \neq 0$, ce qui est absurde, par hypothèse, et qui achève la démonstration. 
Remarque 2.5. - En particulier, si $\pi$ est une représentation cuspidale, il existe une unique $\pi^{\prime}$ (l'unique quotient irréductible de $\sharp-\operatorname{ind}_{P_{m-n, n}^{\prime}}^{G_{m}^{\prime}}\left(1_{m-n} \otimes \widetilde{\pi}\right)$ ), telle que

$$
\operatorname{Hom}_{G_{n} \times G_{m}^{\prime}}\left(\sigma_{n, m}, \pi \otimes \pi^{\prime}\right) \neq 0 .
$$

En effet, si $\pi$ est cuspidale, il n'existe pas $\tau \in \operatorname{Irr}\left(G_{k}\right), k<n$, telle que

$$
\operatorname{Hom}_{G_{n}}\left(\sharp-\operatorname{ind}_{\bar{P}_{n-k ; k}}^{G_{n}}\left(1_{n-k} \otimes \tau\right), \pi\right) \neq 0,
$$

et donc, par le corollaire 2.3, elle n'apparaît pas dans le bord de $\sigma_{n, m}$.

\section{Suite de Kudla}

Dans cette section, on refait les calculs de [11], pour les paires duales de type II. On veut calculer une suite de composition des foncteurs de Jacquet de la représentation $\sigma_{n, m}$.

Soient $t, i$ des entiers, $0<t \leq n, 0 \leq i \leq \inf \{t, m\}$. Fixons quelques notations pour cette section :

- On notera chaque matrice $\mathbf{m} \in \mathcal{M}_{n, m}$

$$
\mathbf{m}=\left(\begin{array}{l}
a \\
b
\end{array}\right)=\left(\begin{array}{c}
a \\
m_{1} m_{2}
\end{array}\right),
$$

où $a \in \mathcal{M}_{t, m}, b \in \mathcal{M}_{n-t, m}, m_{1} \in \mathcal{M}_{n-t, i}, m_{2} \in \mathcal{M}_{n-t, m-i}$, .

- On notera chaque $g \in M_{(t, n-t)}$

$$
g=\left(\begin{array}{ll}
g_{0} & \\
& g_{1}
\end{array}\right)=\left(\begin{array}{lll}
g_{2} & g_{3} & \\
g_{4} & g_{5} & \\
& & g_{1}
\end{array}\right),
$$

$g_{0} \in G_{t}, g_{1} \in G_{n-t}, g_{2} \in \mathcal{M}_{t-i, t-i}, g_{3} \in \mathcal{M}_{t-i, i}, g_{4} \in \mathcal{M}_{i, t-i}, g_{5} \in \mathcal{M}_{i, i}$.

- On notera chaque $g^{\prime} \in G_{m}^{\prime}$

$$
g^{\prime}=\left(\begin{array}{ll}
g_{1}^{\prime} & g_{2}^{\prime} \\
g_{3}^{\prime} & g_{4}^{\prime}
\end{array}\right)
$$

où $g_{1}^{\prime} \in \mathcal{M}_{i, i}, g_{2}^{\prime} \in \mathcal{M}_{m-i, i}, g_{3}^{\prime} \in \mathcal{M}_{i, m-i}, g_{4}^{\prime} \in \mathcal{M}_{m-i, m-i}$.

Soit $\psi$ un caractère non trivial de $F$.

On définit $\sigma_{n, m}^{*}$ par le diagramme commutatif suivant :

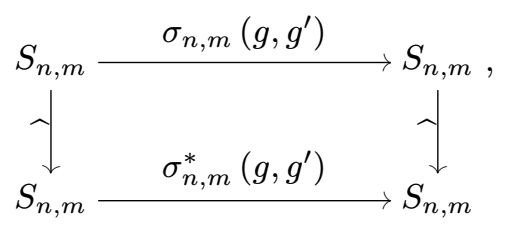

où est l'isomorphisme de représentations qui envoie $f \in S_{n, m}$ vers

$$
\widehat{f}\left(\begin{array}{l}
a \\
b
\end{array}\right)=\int_{\mathcal{M}_{t, m}} f\left(\begin{array}{c}
a^{*} \\
b
\end{array}\right) \psi \circ \operatorname{trd}\left({ }^{t} a a^{*}\right) d a^{*},
$$

où on a noté trd la trace réduite. 
On se propose d'étudier la représentation $\sigma_{n, m}^{*}$ (transformée de Fourier partielle de $\sigma_{n, m}$, isomorphe à $\sigma_{n, m}$ par (3.1)). Plus tard, on ne fera pas de distinction entre ces deux représentations.

La représentation $\sigma_{n, m}^{*}$ agit sur $\widehat{f}$ par

$$
\sigma_{n, m}^{*}\left(g, g^{\prime}\right) \widehat{f}\left(\begin{array}{l}
a \\
b
\end{array}\right)=\int_{\mathcal{M}_{n, t}} f\left(g^{-1}\left(\begin{array}{l}
a^{*} \\
b
\end{array}\right) g^{\prime}\right) \psi \circ \operatorname{trd}\left({ }^{t} a a^{*}\right) d a^{*} .
$$

Ainsi $U_{t, n-t}$ agit, via $\sigma_{n, m}^{*}$, par

$$
\begin{aligned}
\sigma_{n, m}^{*}\left(\begin{array}{rr}
1 & u \\
1
\end{array}\right) \widehat{f}\left(\begin{array}{l}
a \\
b
\end{array}\right) & =\psi \circ \operatorname{trd}\left({ }^{t} a u b\right) \widehat{f}\left(\begin{array}{l}
a \\
b
\end{array}\right) \\
& =\psi \circ \operatorname{trd}\left(b^{t} a u\right) \hat{f}\left(\begin{array}{l}
a \\
b
\end{array}\right) .
\end{aligned}
$$

Notons $A$ la partie fermée de $\mathcal{M}_{n, m}$ :

$$
A=\left\{\left(\begin{array}{l}
a \\
b
\end{array}\right) \in \mathcal{M}_{n, m}: b^{t} a=0\right\} .
$$

Lemme 3.1. - Le sous-espace $S_{n, m}\left(U_{t, n-t}, \sigma_{n, m}^{*}\right)$ de $S_{n, m}$ engendré par les fonctions de la forme

$$
\widehat{f}-\sigma_{n, m}^{*}(u) \widehat{f}
$$

où $f \in S_{n, m}$ et $u \in U_{t, n-t}$, est l'espace des $\widehat{f} \in S_{n, m}$ telles que

$$
\operatorname{supp} \widehat{f} \cap A=\varnothing \text {. }
$$

Démonstration. - Sur $A, \widehat{f}-\sigma_{n, m}^{*}(u) \widehat{f}$ est, par (3.3), nul. Réciproquement, soit $\widehat{f} \neq 0$ nulle sur $A$; montrons que $\widehat{f} \in S_{n, m}\left(U_{t, n-t}, \sigma_{n, m}^{*}\right)$. Le lemme sera démontré.

Pour tout $\mathbf{m}=\left(\begin{array}{l}a \\ b\end{array}\right) \in \mathcal{M}_{n, m}$, notons $\alpha_{\mathbf{m}}$ le caractère défini

$$
\begin{aligned}
\alpha_{\mathbf{m}}: U_{t, n-t} & \rightarrow \mathbb{C} \\
u & \mapsto \psi \circ \operatorname{trd}\left({ }^{t} a u b\right) .
\end{aligned}
$$

Pour tout $\mathbf{m} \in \mathcal{M}_{n, m} \backslash A$, il existe $u_{\mathbf{m}} \in U_{t, n-t}$ tel que $\alpha_{\mathbf{m}}\left(u_{\mathbf{m}}\right) \neq 1$. Pour $\mathbf{m}^{\prime}$ dans un voisinage $I$ de $\mathbf{m}$, on a par continuité $\alpha_{\mathbf{m}^{\prime}}\left(u_{\mathbf{m}}\right)=\alpha_{\mathbf{m}}\left(u_{\mathbf{m}}\right) \neq 1$. Le support de $\widehat{f}$, étant compact, il suffit de montrer que la fonction caractéristique $\mathbf{1}_{I}$ du voisinage $I$ est de la forme $\widehat{F}-\sigma_{n, m}^{*}(u) \widehat{F}$, pour $F \in S_{n, m}$.

Puisque $\alpha_{\mathbf{m}^{\prime}}\left(u_{\mathbf{m}}\right)=\alpha_{\mathbf{m}}\left(u_{\mathbf{m}}\right) \neq 1$, on a que $\mathbf{1}_{I}$ est égal à $\sigma_{n, m}^{*}(u) \mathbf{1}_{I}$ à une constante non nulle près. Ainsi, $\widehat{F}=\frac{1}{1-\alpha_{\mathbf{m}}\left(u_{\mathbf{m}}\right)} \mathbf{1}_{I}$ convient.

Ainsi la suite exacte courte $(c f .[2, \S 1.1 .8])$

$$
\begin{aligned}
0 \rightarrow S(\mathcal{M} \backslash A) \rightarrow S_{n, m} & \rightarrow S(A) \rightarrow 0 \\
\widehat{f} & \left.\mapsto \widehat{f}\right|_{A}
\end{aligned}
$$

s’identifie à

$$
0 \rightarrow S_{n, m}\left(U_{t, n-t}, \sigma_{n, m}^{*}\right) \rightarrow S_{n, m} \rightarrow S(A) \rightarrow 0
$$

$4^{\text {e }}$ SÉRIE - TOME $41-2008-\mathrm{N}^{\circ} 5$ 
et donc $S(A)$, muni de l'action de $M_{(t, n-t)}$ donnée par (3.2), s'identifie au foncteur de Jacquet non normalisé, d'où un isomorphisme de $M_{(t, n-t)}$-modules :

$$
S(A) \simeq \sharp-r_{t, n-t}^{G_{n}}\left(\sigma_{n, m}\right) .
$$

On a alors une filtration de $\sharp-r_{t, n-t}^{G_{n}}\left(\sigma_{n, m}\right)$ de $M_{(t, n-t)} \times G_{m}^{\prime}$-modules :

$$
0=S_{k+1} \subset S_{k} \subset \cdots \subset S_{1} \subset S_{0}=\sharp-r_{t, n-t}^{G_{n}}\left(\sigma_{n, m}\right),
$$

où

$$
\begin{aligned}
S_{i} & =\left\{\widehat{f} \in S(A): \widehat{f}\left(\begin{array}{l}
a \\
b
\end{array}\right)=0 \text { si } \operatorname{rang}(a) \leq i-1\right\} \\
k & =\inf (t, m) .
\end{aligned}
$$

Chaque $S_{i+1}$ est ouvert dans $S_{i}$. La représentation $\sharp-r_{t, n-t}^{G_{n}}\left(\sigma_{n, m}\right)$ est donc composée des représentations

$$
\tau_{i}^{*}=S_{i} / S_{i+1} \quad 0 \leq i \leq k .
$$

La suite exacte de $M_{(t, n-t)} \times G_{m}^{\prime}$-modules $(c f .[2, \S 1.1 .8])$

$$
0 \rightarrow S_{i} \rightarrow S_{i+1} \rightarrow S\left(A_{i}\right) \rightarrow 0
$$

où $A_{i}=\left\{\left(\begin{array}{l}a \\ b\end{array}\right) \in \mathcal{M}_{n, m}: b^{t} a=0\right.$ et $\left.\operatorname{rang}(a)=i\right\}$, nous montre que l'espace de $\tau_{i}^{*}$ est l'espace des fonctions $\left\{\widehat{f} \in S\left(A_{i}\right)\right\}$ et $M_{(t, n-t)} \times G_{m}^{\prime}$ agit, via $\tau_{i}^{*}$, sur cet espace par (3.2).

Maintenant on va appliquer le lemme 1.3 , avec $X=A_{i}$ et $X^{\prime}$ l'ensemble des matrices de la forme $\left(\begin{array}{c}a_{i} \\ 0 *\end{array}\right)$, avec $* \in \mathcal{M}_{n-t, m-i}$. Le stabilisateur $T_{i}$ dans $M_{(t, n-t)} \times G_{m}^{\prime}$ de $X^{\prime}$ est le sous-groupe des $\left(g, g^{\prime}\right) \in P_{t-i, i} \times G_{n-t} \times P_{i, m-i}^{\prime}$ tels que $g_{5}^{-1} g_{1}^{\prime}=1$. Clairement $M_{(t, n-t)} \times G_{m}^{\prime} \cdot X^{\prime}=A_{i}$. Voyons que l'action de $T_{i}$ dans $S\left(X^{\prime}\right)$ est isomorphe à $\xi_{t, i}^{0} \otimes \sigma_{n-t, m-i}$ où $\xi_{t, i}^{0}$ est le caractère de $T_{i}$ défini par $\nu\left(g_{0}\right)^{m} \nu\left(g^{\prime}\right)^{-t}$, pour $\left(g, g^{\prime}\right) \in T_{i}$.

Soient $\left(g, g^{\prime}\right) \in T_{i}, x \in S_{n-t, m-i}$, et $\widehat{f} \in S\left(A_{i}\right)$. On a que

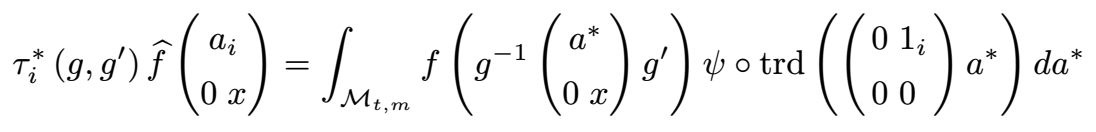

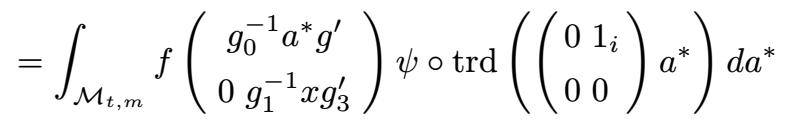

$$
\begin{aligned}
& =\nu\left(g_{0}\right)^{m} \nu\left(g^{\prime}\right)^{-t} \int_{\mathcal{M}_{t, m}} f\left(\begin{array}{c}
a^{*} \\
0 g_{1}^{-1} x g_{3}^{\prime}
\end{array}\right) \psi \circ \operatorname{trd}\left(\left(\begin{array}{cc}
0\left(g_{5}^{-1} g_{1}^{\prime}\right)^{-1} \\
0 & 0
\end{array}\right) a^{*}\right) d a^{*} \\
& =\nu\left(g_{0}\right)^{m} \nu\left(g^{\prime}\right)^{-t} \int_{\mathcal{M}_{t, m}} f\left(\begin{array}{c}
a^{*} \\
0 g_{1}^{-1} x g_{3}^{\prime}
\end{array}\right) \psi \circ \operatorname{trd}\left(\left(\begin{array}{ll}
0 & 1 \\
0 & 0
\end{array}\right) a^{*}\right) d a^{*} \\
& =\left.\nu\left(g_{0}\right)^{m} \nu\left(g^{\prime}\right)^{-t} \sigma_{n-t, m-i}\left(g_{1}, g_{3}^{\prime}\right) \widehat{f}\right|_{\left(\begin{array}{c}
a_{i} \\
0 *
\end{array}\right)}(x) \text {. }
\end{aligned}
$$


Proposition 3.2. - La représentation $r_{t, n-t}^{G_{n}}\left(\sigma_{n, m}\right)$ est composée des représentations $\tau_{i}$, $i=0, \ldots, \min \{t, m\}$, ò̀

$$
\tau_{i} \simeq \operatorname{ind}_{P_{t-i, i} \times G_{n-t} \times P_{i, m-i}^{\prime}}^{M_{(t, n-t)} \times G_{t, i}^{\prime}}\left(\xi_{t} \otimes \rho_{i} \otimes \sigma_{n-t, m-i}\right),
$$

et où $\rho_{i}$ est définie par (2.2) et $\xi_{t, i}$ est le caractère

$$
\xi_{t, i}= \begin{cases}\nu^{\frac{2 m-n+t-i}{2}} & \text { sur } G_{t-i} \\ \nu^{\frac{2 m-n+2 t-i}{2}} & \text { sur } G_{i} \\ \nu^{\frac{t}{2}} & \text { sur } G_{n-t} \\ \nu \frac{-m-2 t+i}{2} & \text { sur } G_{i}^{\prime} \\ \nu \frac{-2 t+i}{2} & \text { sur } G_{m-i}^{\prime}\end{cases}
$$

Démonstration. - D’après le lemme 1.3, ce qui précède implique que

$$
\tau_{i}^{*} \simeq \sharp-\operatorname{ind}_{T_{i}}^{M_{(t, n-t)} \times G_{m}^{\prime}}\left(\xi_{t, i}^{0} \otimes \sigma_{n-t, m-i}\right) .
$$

Induire de $T_{i}$ à $M_{(t, n-t)} \times G_{m}^{\prime}$ revient à induire de $T_{i}$ à $P_{t-i, i} \times G_{n-t} \times P_{i, m-i}$, puis à $M_{(t, n-t)} \times G_{m}^{\prime}$. Or, l'induite

$$
\sharp-\operatorname{ind}_{T_{i}}^{P_{t-i, i} \times G_{n-t} \times P_{i, m-i}^{\prime}}\left(\xi_{t, i}^{0} \otimes \sigma_{n-t, m-i}\right)
$$

est la représentation $\xi_{t, i}^{0} \otimes \rho_{i} \otimes \sigma_{n-t, m-i}$.

Ainsi, l'induite $\sharp-\operatorname{ind}_{T_{i}}^{M_{(t, n-t)} \times G_{m}^{\prime}}\left(\xi_{t, i}^{0} \otimes \sigma_{n-t, m-i}\right)$ est la représentation

$$
\sharp-\operatorname{ind}_{P_{t-i, i} \times G_{n-t} \times P_{i, m-i}^{\prime}}^{M_{(t, n-t)} \times G_{m}^{\prime}}\left(\xi_{t, i}^{0} \otimes \rho_{i} \otimes \sigma_{n-t, m-i}\right) .
$$

Pour achever la proposition, il ne nous reste qu'à changer les induites et foncteurs de Jacquet non normalisés en induites et foncteurs de Jacquet normalisés. Ainsi

$$
\tau_{i} \simeq \delta_{P_{t, n-t}}^{-\frac{1}{2}} \tau_{i}^{*} \simeq \operatorname{ind}_{P_{t-i, i} \times G_{n-t} \times P_{i, m-i}^{\prime}}^{M_{(t, n-t)} \times G_{t, i}^{\prime}}\left(\xi_{t} \otimes \rho_{i} \otimes \sigma_{n-t, m-i}\right),
$$

avec

$$
\xi_{t, i}=\nu\left(g_{0}\right)^{m} \nu\left(g^{\prime}\right)^{-t} \delta_{P_{t-i, i}}^{\frac{-1}{2}} \delta_{P_{t, n-t}}^{\frac{-1}{2}} \delta_{P_{i, m-i}^{\prime}}^{\frac{-1}{2}},
$$

i.e. le caractère requis.

Avec les mêmes arguments, on calcule une suite de composition du foncteur de Jacquet $\bar{r}_{t, m-t}^{G_{m}^{\prime}}$, agissant cette fois-ci du côté de $G_{m}^{\prime}$.

Proposition 3.3. - Soient $t, i$ des entiers, $0<t \leq m 0 \leq i \leq \inf \{t, n\}$. La représentation $\bar{r}_{t, m-t}^{G_{m}^{\prime}}\left(\sigma_{n, m}\right)$ est composée des représentations ${\overline{\tau^{\prime}}}_{i}, i=0, \ldots, \min \{t, n\}$ où

$$
{\overline{\tau^{\prime}}}_{i} \simeq \operatorname{ind}_{P_{n-i, i} \times P_{i, t-i}^{\prime} \times G_{m-t}^{\prime}}^{G_{n} \times M_{(t, m-t)}^{\prime}}\left(\sigma_{n-i, m-t} \otimes \rho_{i} \otimes{\overline{\xi^{\prime}}}_{t, i}\right)
$$


et où $\rho_{i}$ est définie par (2.2) et ${\overline{\xi^{\prime}}}_{t, i}$ est le caractère

$$
\bar{\xi}_{t, i}^{\prime}= \begin{cases}\nu^{\frac{2 t-i}{2}} & \text { sur } G_{n-i} \\ \nu^{\frac{n+2 t-i}{2}} & \text { sur } G_{i} \\ \nu^{\frac{-2 n+m-2 t+i}{2}} & \text { sur } G_{i}^{\prime} \\ \nu^{\frac{m-2 n-t+i}{2}} & \text { sur } G_{t-i}^{\prime} \\ \nu^{\frac{-t}{2}} & \text { sur } G_{m-t}^{\prime} .\end{cases}
$$

\section{Application}

Soient $\pi \in \operatorname{Irr}\left(G_{n}\right), \pi^{\prime} \in \operatorname{Irr}\left(G_{m}^{\prime}\right)$ telles que $\pi \otimes \pi^{\prime}$ soit un quotient de $\sigma_{n, m}$. Soit $r$ un entier positif. Pour toute représentation cuspidale $\chi$ de $G_{r}$, considérons l'entier positif maximal $a$ tel que $\pi$ soit une sous-représentation d'une représentation de la forme

$$
\chi \times \chi \times \cdots \times \chi \times \rho,
$$

où on a fait le produit de $a$ fois la représentation $\chi$ fois $\rho, \rho$ étant une représentation irréductible de $G_{n-r a}$. Alors, par exactitude du foncteur de Jacquet, on a un entrelacement surjectif de $r_{r a, n-r a}^{G_{n}}\left(\sigma_{n, m}\right)$ dans $r_{r a, n-r a}^{G_{n}}(\pi) \otimes \pi^{\prime}$, d'où un entrelacement non nul de $r_{r a, n-r a}^{G_{n}}\left(\sigma_{n, m}\right)$ dans $\chi \times \chi \times \cdots \times \chi \otimes \rho \otimes \pi^{\prime}$.

D'après 3.2, il existe alors $i \in\{0, \ldots, r a\}$ tel que

$$
\operatorname{Hom}\left(\tau_{i}, \chi \times \chi \times \cdots \times \chi \otimes \rho \otimes \pi^{\prime}\right) \neq 0 .
$$

Lemme 4.1. - Les seuls $\tau_{i}$ qui peuvent avoir des quotients de la forme ci-dessus sont $\tau_{r a}$ et $\tau_{\text {ra-1 }}$ et, si $\chi \neq \nu^{\frac{2 m-n+1}{2}}$, seul $\tau_{\text {ra }}$ peut en avoir.

Démonstration. - En effet, supposons que

$$
\operatorname{Hom}\left(\tau_{i}, \chi \times \chi \times \cdots \times \chi \otimes \rho \otimes \pi^{\prime}\right) \neq 0 .
$$

Cela signifie, par définition de $\tau_{i}$,

$\operatorname{Hom}\left(\operatorname{ind}_{P_{r a-i, i} \times G_{n-r a} \times P_{i, m-i}^{\prime}}^{M_{(r a, n-r a} \times G_{m}^{\prime}}\left(\xi_{r a, i} \otimes \rho_{i} \otimes \sigma_{n-r a, m-i}\right), \chi \times \chi \times \cdots \times \chi \otimes \rho \otimes \pi^{\prime}\right) \neq 0$

et, par (1.2),

$$
\operatorname{Hom}\left(\xi_{r a, i} \otimes \rho_{i} \otimes \sigma_{n-r a, m-i}, \bar{r}_{P_{r a-i, i} \times G_{n-r a} \times P_{i, m-i}^{\prime}}^{M_{(r a, n-r a} \times G_{m}^{\prime}}\left(\chi \times \chi \times \cdots \times \chi \otimes \rho \otimes \pi^{\prime}\right)\right) \neq 0,
$$

d'où

$$
\operatorname{Hom}\left(\xi_{r a, i} \otimes \rho_{i} \otimes \sigma_{n-r a, m-i}, \bar{r}_{P_{r a-i, i}}^{G_{r a}}(\chi \times \chi \times \cdots \times \chi) \otimes \rho \otimes \bar{r}_{P_{i, m-i}^{\prime}}^{G_{m}^{\prime}}\left(\pi^{\prime}\right)\right) \neq 0,
$$

et donc

$$
\operatorname{Hom}\left(\left.\xi_{r a, i}\right|_{G_{r a-i}},\left.\bar{r}_{P_{r a-i, i}}^{G_{r a}}(\chi \times \chi \times \cdots \times \chi)\right|_{G_{r a-i}}\right) \neq 0 .
$$

Ainsi, on trouve finalement

$$
\operatorname{Hom}\left(\nu_{r a-i}^{\frac{2 m-n+r a-i}{2}}, \chi \times \chi \times \cdots \times \chi\right) \neq 0 .
$$

Par l'unicité du support cuspidal, il faut alors que

$$
\operatorname{supp}\left(\nu_{r a-i}^{\frac{2 m-n+r a-i}{2}}\right)=\{\chi, \ldots, \chi\}
$$


et donc, ou bien $i=r a$, ou bien $i=r a-1$ et $\chi=\nu^{\frac{2 m-n+1}{2}}$.

Ainsi on se retrouve avec deux cas :

CAS A, Hom $\left(\tau_{r a}, \chi \times \chi \times \cdots \times \chi \otimes \rho \otimes \pi^{\prime}\right) \neq 0$,

$\mathrm{CAS} \mathrm{B}, \operatorname{Hom}\left(\tau_{r a-1}, \chi \times \chi \times \cdots \times \chi \otimes \rho \otimes \pi^{\prime}\right) \neq 0$ et $\chi=\nu^{\frac{2 m-n+1}{2}}$.

Examinons successivement les différents cas du lemme 4.1 (le cas B sera traité dans la section 9).

CAS A. Supposons d'abord Hom $\left(\tau_{r a}, \chi \times \chi \times \cdots \times \chi \otimes \rho \otimes \pi^{\prime}\right) \neq 0$ (ce qui arrive, en particulier, d'après le lemme précédent, pour $\chi$ distinct de $\nu^{\frac{2 m-n+1}{2}}$ ).

Alors, d'après la proposition 3.2

$$
\begin{aligned}
\text { Hom }\left(\operatorname{ind}_{M_{(r a, n-r a)} \times P_{r a, m-r a}^{\prime}}^{M_{(r a, n-r a} \times G_{m}^{\prime}}\left(\xi_{r a, r a} \otimes \rho_{r a} \otimes \sigma_{n-r a, m-r a}\right),\right. & \\
& \left.\chi \times \chi \times \cdots \times \chi \otimes \rho \otimes \pi^{\prime}\right) \neq 0 .
\end{aligned}
$$

Pour simplifier les notations, à la place de ind $M_{(r a, n-r a)}^{M_{(r a, n a)} \times G_{m}^{\prime}} P_{r a, m-r a}^{\prime}$, on écrira ind $\operatorname{ind}_{P_{r a, m-r a}^{\prime}}^{G_{m}^{\prime}}$, l'induction du côte de $M_{(r a, n-r a)}$ étant triviale (même si on considère la représentation $\operatorname{ind}_{P_{r a, m-r a}^{\prime}}^{G_{m}^{\prime}}\left(\rho_{r a} \otimes \sigma_{n-r a, m-r a}\right)$ en tant que $M_{(r a, n-r a)} \times G_{m}^{\prime}$-représentation).

Ainsi, l'inégalité (4.1) s'écrit, par définition de $\xi_{r a, r a}$

$$
\begin{aligned}
\operatorname{Hom}\left(\operatorname{ind}_{P_{r a, m-r a}^{\prime}}^{G_{m}^{\prime}}\left(\rho_{r a} \nu^{\prime \frac{m}{2}} \otimes \sigma_{n-r a, m-r a}\right)\right. & \\
& \left.\nu^{\frac{n}{2}} \chi \times \nu^{\frac{n}{2}} \chi \times \cdots \times \nu^{\frac{n-m}{2}} \chi \otimes \nu^{\frac{-r a}{2}} \rho \otimes \nu^{\frac{r a}{2}} \pi^{\prime}\right) \neq 0
\end{aligned}
$$

d'où, par le lemme [14, 3.II.3],

$$
\operatorname{Hom}\left(\operatorname{ind}_{P_{r a, m-r a}^{\prime}}^{G_{m}^{\prime}}\left(\nu^{\frac{m-n}{2}} \tilde{\chi} \times \cdots \times \nu^{\frac{m-n}{2}} \tilde{\chi} \otimes \nu^{\frac{r a}{2}} \sigma_{n-r a, m-r a} \nu^{\frac{-r a}{2}}\right), \rho \otimes \pi^{\prime}\right) \neq 0 .
$$

Soit $b \geq 0$ maintenant maximal tel qu'il existe une représentation irréductible $\rho^{\prime} \operatorname{de} G_{m-r b}^{\prime}$ avec $\pi^{\prime}$ quotient de

$$
\nu^{\frac{m-n}{2}} \tilde{\chi} \times \cdots \times \nu^{\frac{m-n}{2}} \tilde{\chi} \times \rho^{\prime}
$$

où on a fait le produit de $b$ fois la représentation $\nu^{\frac{m-n}{2}} \tilde{\chi}$.

Ceci équivaut, par le corollaire 1.2, au fait que $\pi^{\prime}$ soit sous-module de

$$
\rho^{\prime} \times \nu^{\frac{m-n}{2}} \tilde{\chi} \times \cdots \times \nu^{\frac{m-n}{2}} \tilde{\chi}
$$

où on a fait le produit de $b$ fois la représentation $\nu^{\frac{m-n}{2}} \tilde{\chi}$.

Par (1.1), on a un homomorphisme non trivial de $r_{m-r b, r b}^{G_{m}^{\prime}}\left(\pi^{\prime}\right)$ dans $\rho^{\prime} \otimes \nu^{\frac{m-n}{2}} \widetilde{\chi} \times$ $\cdots \times \nu^{\frac{m-n}{2}} \tilde{\chi}$ d'où, par conjugaison, un homomorphisme non trivial de $\bar{r}_{r b, m-r b}^{G_{m}^{\prime}}\left(\pi^{\prime}\right)$ dans $\nu^{\frac{m-n}{2}} \tilde{\chi} \times \cdots \times \nu^{\frac{m-n}{2}} \tilde{\chi} \otimes \rho^{\prime}$.

D'un autre côté, le foncteur de Jacquet étant exact, on a un morphisme surjectif dans

$$
\begin{gathered}
\operatorname{Hom}\left(\bar{r}_{r b, m-r b}^{G_{m}^{\prime}} \circ \operatorname{ind}_{P_{r a, m-r a}^{\prime}}^{G_{m}^{\prime}}\left(\nu^{\frac{m-n}{2}} \tilde{\chi} \times \ldots \times \nu^{\frac{m-n}{2}} \tilde{\chi} \otimes \nu^{\frac{r a}{2}} \sigma_{n-r a, m-r a} \nu^{\prime-r a} \frac{r}{2}\right),\right. \\
\left.\rho \otimes \bar{r}_{r b, m-r b}^{G_{m}^{\prime}}\left(\pi^{\prime}\right)\right)
\end{gathered}
$$


qui, composé avec l'homomorphisme non trivial précédent de $\bar{r}_{r b, m-r b}^{G_{m}^{\prime}}\left(\pi^{\prime}\right)$ dans $\nu^{\frac{m-n}{2}} \widetilde{\chi} \times$ $\cdots \times \nu^{\frac{m-n}{2}} \tilde{\chi} \otimes \rho^{\prime}$, montre que

$$
\begin{gathered}
\operatorname{Hom}\left(\bar{r}_{r b, m-r b}^{G_{m}^{\prime}} \circ \operatorname{ind}_{P_{r a, m-r a}^{\prime}}^{G_{m}^{\prime}}\left(\nu^{\frac{m-n}{2}} \tilde{\chi} \times \ldots \times \nu^{\frac{m-n}{2}} \tilde{\chi} \otimes \nu^{\frac{r a}{2}} \sigma_{n-r a, m-r a} \nu^{\frac{{ }^{-r a}}{2}}\right)\right. \\
\left.\rho \otimes \nu^{\frac{m-n}{2}} \tilde{\chi} \times \cdots \times \nu^{\frac{m-n}{2}} \tilde{\chi} \otimes \rho^{\prime}\right) \neq 0
\end{gathered}
$$

Par le lemme géométrique ( $c f .[21, \S 1.6])$ et la maximalité de $b$, on déduit que :

$$
\begin{gathered}
\operatorname{Hom}\left(\operatorname{ind}_{P_{r a, r b-r a}^{\prime}}^{G_{r b}^{\prime}}\left(\nu^{\frac{m-n}{2}} \tilde{\chi} \times \ldots \times \nu^{\frac{m-n}{2}} \tilde{\chi} \otimes \nu^{\frac{r a}{2}} \bar{r}_{r b-r a, m-r b}^{G_{m-r a}^{\prime}}\left(\sigma_{n-r a, m-r a}\right) \nu^{\prime \frac{-r a}{2}}\right)\right. \\
\left.\rho \otimes \nu^{\frac{m-n}{2}} \tilde{\chi} \times \cdots \times \nu^{\frac{m-n}{2}} \tilde{\chi} \otimes \rho^{\prime}\right) \neq 0 .
\end{gathered}
$$

D'après la proposition 3.3, il existe alors $i \in\{0, \ldots, r b-r a\}$ tel que

$$
\operatorname{Hom}\left(\overline{\tau_{i}^{\prime}}, \nu^{\frac{m-n+r a}{2}} \tilde{\chi} \times \cdots \times \nu^{\frac{m-n+r a}{2}} \tilde{\chi} \otimes \nu^{\frac{r a}{2}} \rho^{\prime}\right) \neq 0 .
$$

Le lemme suivant se montre comme le lemme 4.1 :

Lemme 4.2. - Les seuls $\overline{\tau^{\prime}}{ }_{i}$ qui peuvent avoir des quotients de la forme ci-dessus sont ${\overline{\tau^{\prime}}}_{r b-r a}$ et ${\overline{\tau^{\prime}}}_{r b-r a-1}$. Or, si $\nu^{\frac{m-n}{2}} \tilde{\chi} \neq \nu^{\frac{m-2 n-1}{2}}$, (i.e. si $\chi \neq \nu^{\frac{n+1}{2}}$ ) seul ${\overline{\tau^{\prime}}}_{r b-r a}$ peut en avoir.

Ainsi on a, à nouveau, deux cas :

CAS A.1. Hom $\left({\overline{\tau^{\prime}}}_{r b-r a}, \nu^{\frac{m-n+r a}{2}} \tilde{\chi} \times \cdots \times \nu^{\frac{m-n+r a}{2}} \tilde{\chi} \otimes \nu^{\frac{r a}{2}} \rho^{\prime}\right) \neq 0$ (ce qui arrive, en particulier, d'après le lemme précédent si $\chi \neq \nu^{\frac{2 m-n+1}{2}}$ et $\chi \neq \nu^{\frac{n+1}{2}}$ ).

CAS A.2. Hom $\left({\overline{\tau^{\prime}}}_{r b-r a-1}, \nu^{\frac{m-n+r a}{2}} \tilde{\chi} \times \cdots \times \nu^{\frac{m-n+r a}{2}} \tilde{\chi} \otimes \nu^{\frac{r a}{2}} \rho^{\prime}\right) \neq 0$ et, dans ce cas, il faut que $\chi=\nu^{\frac{n+1}{2}}$.

Regardons d'abord le cas A.1.

Lemme 4.3. - Dans le cas A.1, on $a b=a$.

Démonstration. - Si

$$
\begin{gathered}
\operatorname{Hom}\left(\operatorname{ind}_{P_{r a, r b-r a}^{\prime}}^{G_{r b}^{\prime}}\left(\nu^{\frac{m-n}{2}} \tilde{\chi} \times \cdots \times \nu^{\frac{m-n}{2}} \tilde{\chi} \otimes \nu^{\frac{r a}{2}}{\overline{\tau^{\prime}}}_{r b-r a} \nu^{\prime \frac{-r a}{2}}\right),\right. \\
\left.\rho \otimes \nu^{\frac{m-n}{2}} \tilde{\chi} \times \cdots \times \nu^{\frac{m-n}{2}} \tilde{\chi} \otimes \rho^{\prime}\right) \neq 0
\end{gathered}
$$

alors, par définition de ${\overline{\tau^{\prime}}}_{r b-r a}$, on a aussi

$$
\begin{gathered}
\operatorname{Hom}\left(\operatorname{ind}_{P_{n-r b, r b-r a}}^{G_{n-r a}}\left(\nu^{\frac{r b-r a}{2}} \sigma_{n-r b, m-r b} \nu^{\frac{-r b+r a}{2}} \otimes \nu^{\frac{-r a}{2}} \chi \times \cdots \times \nu^{\frac{-r a}{2}} \chi\right),\right. \\
\left.\nu^{\frac{-r a}{2}} \rho \otimes \nu^{\frac{r a}{2}} \rho^{\prime}\right) \neq 0
\end{gathered}
$$

où on a fait le produit de $b-a$ fois la représentation $\nu^{\frac{-r a}{2}} \chi$, et donc, par maximalité de $a$, il faut que $b=a$.

Avant de passer aux autres cas, résumons les résultats obtenus en une proposition. 
Proposition 4.4. - Soient $\pi \in \operatorname{Irr}\left(G_{n}\right), \pi^{\prime} \in \operatorname{Irr}\left(G_{m}^{\prime}\right)$ telles que $\pi \otimes \pi^{\prime}$ soit un quotient de $\sigma_{n, m}$. Soit aussi

$$
\chi \neq\left\{\begin{array}{l}
\nu^{\frac{n+1}{2}} \\
\nu^{\frac{2 m-n+1}{2}}
\end{array}\right.
$$

une représentation irréductible cuspidale de $G_{r}$. Alors $a=b$ où a et $b$ sont définis par les conditions suivantes:

1. Il existe $\rho \in \operatorname{Irr}\left(G_{n-r a}\right)$ avec

$$
\pi \hookrightarrow \chi \times \chi \times \cdots \times \chi \times \rho,
$$

où on a fait le produit de a fois la représentation cuspidale $\chi$ et a est maximal.

2. Il existe $\rho^{\prime} \in \operatorname{Irr}\left(G_{m-r b}^{\prime}\right)$ avec

$$
\pi^{\prime} \hookrightarrow \rho^{\prime} \times \nu^{\frac{m-n}{2}} \tilde{\chi} \times \cdots \times \nu^{\frac{m-n}{2}} \tilde{\chi},
$$

où on a fait le produit de b fois la représentation cuspidale $\nu^{\frac{m-n}{2}} \tilde{\chi}$ et b est maximal.

De plus, on a

$$
\operatorname{Hom}\left(\sigma_{n-r a, m-r a}, \nu^{\frac{-r a}{2}} \rho \otimes \nu^{\frac{r a}{2}} \rho^{\prime}\right) \neq 0 .
$$

\section{Unicité}

La proposition précédente, avec le théorème 2.4, nous permet de montrer l'unicité de la correspondance thêta.

THÉORÈme 5.1. - Supposons $n \leq m$. Soit $\pi$ une représentation irréductible de $G_{n}$. Il existe une unique représentation irréductible $\pi^{\prime}$ de $G_{m}^{\prime}$ telle que

$$
\operatorname{Hom}_{G_{n} \times G_{m}^{\prime}}\left(\omega_{n, m}, \pi \otimes \pi^{\prime}\right) \neq 0 .
$$

De plus, $\operatorname{dim}\left(\operatorname{Hom}_{G_{n} \times G_{m}^{\prime}}\left(\omega_{n, m}, \pi \otimes \pi^{\prime}\right)\right)=1$.

Démonstration. - Par récurrence, on peut supposer que le théorème est vrai pour toute paire $\left(G_{i}, G_{j}^{\prime}\right)$, où $i j<n m$. Montrons-le pour la paire $\left(G_{n}, G_{m}^{\prime}\right)$.

Soit $\pi^{\prime} \in \operatorname{Irr}\left(G_{m}^{\prime}\right)$ telles que $\pi \otimes \pi^{\prime}$ soit un quotient de $\sigma_{n, m}$. Montrons que $\pi^{\prime}$ est uniquement déterminée par $\pi$.

CAs 1. Supposons d'abord qu'il existe $\chi \neq\left\{\begin{array}{ll}\nu^{\frac{n+1}{2}} \\ \nu^{\frac{2 m-n+1}{2}}\end{array}\right.$ une représentation cuspidale de $G_{r}$, et $\tau \in \operatorname{Irr}\left(G_{n-r}\right)$ avec $\pi \hookrightarrow \chi \times \tau$.

Soient $a>0$ et $\rho \in \operatorname{Irr}\left(G_{n-r a}\right)$ avec

$$
\pi \hookrightarrow \chi \times \chi \times \cdots \times \chi \times \rho,
$$

où on a fait le produit de $a$ fois la représentation cuspidale $\chi$ et $a$ est maximal.

D'après la proposition précédente, il existe $\rho^{\prime} \in \operatorname{Irr}\left(G_{m-r a}^{\prime}\right)$ avec

$$
\pi^{\prime} \hookrightarrow \rho^{\prime} \times \nu^{\frac{m-n}{2}} \tilde{\chi} \times \cdots \times \nu^{\frac{m-n}{2}} \tilde{\chi},
$$

où on a fait le produit de $a$ fois la représentation cuspidale $\nu^{\frac{m-n}{2}} \widetilde{\chi}$. De plus, on a

$$
\operatorname{Hom}\left(\sigma_{n-r a, m-r a}, \nu^{\frac{-r a}{2}} \rho \otimes \nu^{\frac{r a}{2}} \rho^{\prime}\right) \neq 0 \text {. }
$$

$4{ }^{\mathrm{e}}$ SÉRIE - TOME $41-2008-\mathrm{N}^{\mathrm{o}} 5$ 
Par hypothèse de récurrence, $\rho^{\prime}$ est uniquement déterminée par $\rho$ et, $\operatorname{par}[13$, Théorème 5.1], $\pi^{\prime}$ est l'unique sous-module irréductible de $\rho^{\prime} \times \nu^{\frac{m-n}{2}} \tilde{\chi} \times \cdots \times \nu^{\frac{m-n}{2}} \tilde{\chi}$.

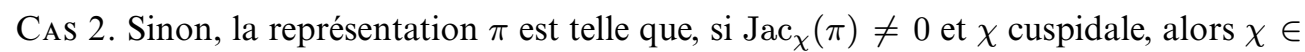
$\left\{\nu^{\frac{n+1}{2}}, \nu^{\frac{2 m-n+1}{2}}\right\}$. Dans ce cas, $\pi$ n'apparaît pas dans le bord de $\sigma_{n, m}$ car elle n'est pas quo-

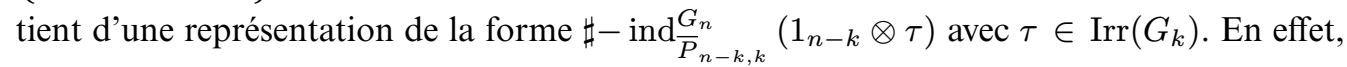
si

on trouve, après normalisation, que

$$
\operatorname{Hom}\left(\sharp-\operatorname{ind} \bar{P}_{n-k, k}^{G_{n}}\left(1_{n-k} \otimes \tau\right), \pi\right) \neq 0,
$$

$$
\operatorname{Hom}\left(\operatorname{ind} \bar{P}_{n-k, k}^{G_{n}}\left(\nu^{\frac{k}{2}} \otimes \nu^{\frac{k-n}{2}} \tau\right), \pi\right) \neq 0 .
$$

Par conjugaison, on déduit

$$
\operatorname{Hom}\left(\operatorname{ind}_{P_{k, n-k}}^{G_{n}}\left(\nu^{\frac{k-n}{2}} \tau \otimes \nu^{\frac{k}{2}}\right), \pi\right) \neq 0
$$

puis, par (1.2),

et, à nouveau par conjugaison,

$$
\operatorname{Hom}\left(\nu^{\frac{k-n}{2}} \tau \otimes \nu^{\frac{k}{2}}, \bar{r}_{P_{k, n-k}}^{G_{n}}(\pi)\right) \neq 0
$$

$$
\operatorname{Hom}\left(\nu^{\frac{k}{2}} \otimes \nu^{\frac{k-n}{2}} \tau, r_{P_{n-k, k}}^{G_{n}}(\pi)\right) \neq 0
$$

et donc Jac ${ }_{\nu} \frac{-n+2 k+1}{2}(\pi) \neq 0$. Ceci n'est possible que si $k=n$ ou $k=m$, ce qui est absurde.

Ainsi d'après 2.4, $\pi^{\prime}$ est l'unique quotient de $\sharp-\operatorname{ind}_{P_{m-n, n}^{\prime}}^{G_{m}^{\prime}}\left(1_{m-n} \otimes \widetilde{\pi}\right)$.

\section{La correspondance explicite}

Soient $\pi \in \operatorname{Irr}\left(G_{n}\right), \pi^{\prime} \in \operatorname{Irr}\left(G_{m}^{\prime}\right)$ telles que

$$
\operatorname{Hom}_{G_{n} \times G_{m}^{\prime}}\left(\omega_{n, m}, \pi \otimes \pi^{\prime}\right) \neq 0 .
$$

La fin de l'article est consacré au calcul des paramètres de Langlands $\pi^{\prime}$ en termes de ceux de $\pi$.

Soient $\tau_{1}, \ldots, \tau_{N}$ des représentations essentiellement de carré intégrable et $\alpha_{1}, \ldots, \alpha_{N} \in \mathbb{R}$ tels que, pour tout $1 \leq i \leq N, \nu^{\alpha_{i}} \tau_{i}$ soit une représentation de carré intégrable. Soit $\sigma$ une permutation de $\{1, \ldots, N\}$ telle que $\alpha_{\sigma(i)} \geq \alpha_{\sigma(j)}$ si $i<j$. La représentation $\tau_{\sigma(1)} \times \tau_{\sigma(2)} \times$ $\cdots \times \tau_{\sigma(N)}$ a un unique quotient irréductible (voir, par exemple, [18, page 54], ou bien [17] pour plus de détails sur la classification de Langlands), et on dira que c'est le quotient de Langlands de $\tau_{1} \times \cdots \times \tau_{N}$.

Supposons que $\pi$ est le quotient de Langlands de $\tau_{1} \times \cdots \times \tau_{N}$, où $\tau_{1}, \ldots, \tau_{N}$ sont des représentations essentiellement de carré intégrable. Notons alors $\theta_{m}^{*}(\pi)$ le quotient de Langlands de

$$
\nu^{\frac{m-2 n-1}{2}} \times \cdots \times \nu^{\frac{-m+1}{2}} \times \nu^{\frac{m-n}{2}} \widetilde{\tau_{1}} \times \cdots \times \nu^{\frac{m-n}{2}} \widetilde{\tau_{N}} .
$$

THÉORÈME 6.1. - Si $m \geq n$ et

$$
\operatorname{Hom}_{G_{n} \times G_{m}^{\prime}}\left(\sigma_{n, m}, \pi \otimes \pi^{\prime}\right) \neq 0,
$$

alors $\pi^{\prime}=\theta_{m}^{*}(\pi)$ 
Remarque 6.2. - En particulier, si $m=n, \pi^{\prime}=\widetilde{\pi}$, comme on l'avait déjà montré au début de la section 2. Dans la preuve, on supposera alors $m>n$.

Des théorèmes 5.1 et 6.1 , il résulte, avec la normalisation correspondante, un théorème similaire pour la représentation $\omega_{n, m}(c f .(1.4))$ :

Corollaire 6.3. - Soit $\pi$ une représentation irréductible de $G_{n}$.

1. Si $\operatorname{Hom}_{G_{n}}\left(\omega_{n, m}, \pi\right) \neq 0$, alors il existe une unique représentation irréductible $\pi^{\prime}$ de $G_{m}^{\prime}$ telle que

$$
\operatorname{Hom}_{G_{n} \times G_{m}^{\prime}}\left(\omega_{n, m}, \pi \otimes \pi^{\prime}\right) \neq 0 .
$$

De plus, $\operatorname{dim}\left(\operatorname{Hom}_{G_{n} \times G_{m}^{\prime}}\left(\omega_{n, m}, \pi \otimes \pi^{\prime}\right)\right)=1$.

2. Supposons $n \leq m$. Alors $\operatorname{Hom}_{G_{n}}\left(\omega_{n, m}, \pi\right) \neq 0$ et, si $\pi$ est le quotient de Langlands de $\tau_{1} \times \cdots \times \tau_{N}$, où $\tau_{1}, \ldots, \tau_{N}$ sont des représentations essentiellement de carré intégrable, alors $\pi^{\prime}$ est le quotient de Langlands de

$$
\nu^{-\frac{m-n-1}{2}} \times \cdots \times \nu^{\frac{m-n-1}{2}} \times \widetilde{\tau_{1}} \times \cdots \times \widetilde{\tau_{N}}
$$

Pour la preuve du théorème 6.1, on va utiliser plusieurs fois le lemme suivant [13, Théorème A.3]

Lemme 6.4. - Soient $\chi$ une représentation cuspidale de $G_{r}$ telle que $\chi \neq\left\{\begin{array}{l}\nu^{\frac{n+1}{2}} \\ \nu^{\frac{2 m-n+1}{2}}\end{array}\right.$ $\rho \in \operatorname{Irr}\left(G_{n-r}\right)$, et $\pi$ l'unique sous-représentation irréductible de $\chi \times \rho$. Notons $\pi^{\prime}$ l'unique sousreprésentation irréductible de $\nu^{\frac{-r}{2}} \theta_{m-r}^{*}\left(\nu^{\frac{-r}{2}} \rho\right) \times \nu^{\frac{m-n}{2}} \widetilde{\chi}$. Alors

$$
\pi^{\prime}=\theta_{m}^{*}(\pi)
$$

Corollaire 6.5. - Soient $\chi$ une représentation cuspidale de $G_{r}, \chi \neq\left\{\begin{array}{l}\nu^{\frac{n+1}{2}} \\ \nu^{\frac{2 m-n+1}{2}} .\end{array}\right.$

Soient $a \in \mathbb{N}^{*}, \rho \in \operatorname{Irr}\left(G_{n-r a}\right)$, et $\pi$ l'unique sous-représentation irréductible de $\chi \times \cdots \times \chi \times \rho$ où on a fait le produit de a fois la représentation $\chi$. Notons $\pi^{\prime}$ l'unique sous-représentation irréductible de $\nu^{\frac{-r a}{2}} \theta_{m-r a}^{*}\left(\nu^{\frac{-r a}{2}} \rho\right) \times \nu^{\frac{m-n}{2}} \tilde{\chi} \times \cdots \times \nu^{\frac{m-n}{2}} \tilde{\chi}$, où on a fait le produit de a fois la représentation $\nu^{\frac{m-n}{2}} \tilde{\chi}$. Alors

$$
\pi^{\prime}=\theta_{m}^{*}(\pi)
$$

Démonstration. - Par récurrence sur $a$. Si $a=1$, c'est le lemme 6.4. Supposons $a>1$. Notons $\pi_{1}$ l'unique sous-représentation irréductible de $\chi \times \cdots \times \chi \times \rho$ où on a fait le produit de $a-1$ fois la représentation $\chi$. Notons $\pi_{1}^{\prime}$ l'unique sous-représentation irréductible de $\nu^{\frac{-r a}{2}} \theta_{m-r a}^{*}\left(\nu^{\frac{-r a}{2}} \rho\right) \times \nu^{\frac{m-n}{2}} \tilde{\chi} \times \cdots \times \nu^{\frac{m-n}{2}} \tilde{\chi}$, où on a fait le produit de $a-1$ fois la représentation $\nu^{\frac{m-n}{2}} \tilde{\chi}$. Alors, par hypothèse de récurrence

$$
\pi_{1}^{\prime}=\nu^{\frac{-r}{2}} \theta_{m-r}^{*}\left(\nu^{\frac{-r}{2}} \rho\right)
$$

et, de plus $\pi$ est l'unique sous-représentation irréductible de $\chi \times \pi_{1}$ et $\pi^{\prime}$ est l'unique sousreprésentation irréductible de $\pi_{1}^{\prime} \times \nu^{\frac{m-n}{2}} \widetilde{\chi}$. Le résultat découle, à nouveau, du lemme 6.4. 


\section{Le cas simple}

Supposons qu'il existe $\chi \neq\left\{\begin{array}{l}\nu^{\frac{n+1}{2}} \\ \nu^{\frac{2 m-n+1}{2}}\end{array} \quad\right.$ une représentation cuspidale de $G_{r}$ et un entier strictement positif $a$, tels que l'une des conditions suivantes équivalentes (par la proposition 4.4) soit satisfaite :

1. Il existe $\rho \in \operatorname{Irr}\left(G_{n-r a}\right)$ avec

$$
\pi \hookrightarrow \chi \times \chi \times \cdots \times \chi \times \rho,
$$

où on a fait le produit de $a$ fois la représentation $\chi, a$ maximal.

2. ou bien, il existe $\rho^{\prime} \in \operatorname{Irr}\left(G_{m-r a}^{\prime}\right)$ avec

$$
\pi^{\prime} \hookrightarrow \rho^{\prime} \times \nu^{\frac{m-n}{2}} \tilde{\chi} \times \cdots \times \nu^{\frac{m-n}{2}} \tilde{\chi},
$$

où on a fait le produit de $a$ fois la représentation $\nu^{\frac{m-n}{2}} \tilde{\chi}, a$ maximal.

Puisque, par la proposition 4.4,

$$
\operatorname{Hom}\left(\sigma_{n-r a, m-r a}, \nu^{\frac{-r a}{2}} \rho \otimes \nu^{\frac{r a}{2}} \rho^{\prime}\right) \neq 0,
$$

et $a>0$, on peut supposer, par hypothèse de récurrence, que

$$
\rho^{\prime}=\nu^{\frac{-r a}{2}} \theta_{m-r a}^{*}\left(\nu^{\frac{-r a}{2}} \rho\right) .
$$

On déduit de (7.1), (7.2) et (7.3), grâce au corollaire 6.5, que $\pi^{\prime}=\theta_{m}^{*}(\pi)$.

\section{Sur les paramètres de Zelevinsky}

Soient $r \in \mathbb{R}, n \in \mathbb{N}^{*}$. On dit que la suite $\Delta=\{r, r+1, \ldots, r+n-1\}$ de nombres réels est un segment et l'ensemble de tous les segments sera noté $S$. Il est muni d'une action de $\mathbb{R}$ définie par

$$
k\{r, r+1, \ldots, r+n-1\}=\{k+r, k+r+1, \ldots, k+r+n-1\} .
$$

On notera aussi

$$
\begin{aligned}
& b(\{r, r+1, \ldots, r+n-1\})=r, \\
& e(\{r, r+1, \ldots, r+n-1\})=r+n-1 .
\end{aligned}
$$

les extrémités du segment. On note $l(\{r, r+1, \ldots, r+n-1\})=n$ sa longueur.

On définit un préordre sur $S$ par $\Delta \leq \Delta^{\prime}$ si $b(\Delta) \leq b\left(\Delta^{\prime}\right)$. On note $M(S)$ l'ensemble de multisegments, i.e. des fonctions $m: S \rightarrow \mathbb{N}$ à support fini (on pensera à un ensemble de segments comptés avec multiplicités). Un multisegment $\Delta_{1}, \Delta_{2}, \ldots, \Delta_{N}$ est dit rangé si $\Delta_{N} \leq \cdots \leq \Delta_{2} \leq \Delta_{1}$.

À chaque segment $\Delta=\{r, r+1, \ldots, r+n-1\}$, on associe une représentation irréductible de $\mathrm{GL}_{n}(D)$, notée $\langle\Delta\rangle^{t}$, définie comme l'unique quotient irréductible de $\nu^{r} \times \nu^{r+1} \times$ $\cdots \times \nu^{r+n-1}$. La représentation $\langle\Delta\rangle^{t}$ est essentiellement de carré intégrable. La contragrédiente $\widetilde{\langle\Delta\rangle^{t}}$ est la représentation $\langle\widetilde{\Delta}\rangle^{t}$ où $\widetilde{\Delta}=\{-r-n+1,-r-n+2, \cdots-r\}$. 
À chaque multisegment $\Delta_{1}, \Delta_{2}, \ldots, \Delta_{N}$, on associe une représentation irréductible de $\operatorname{GL}_{\sum l\left(\Delta_{i}\right)}(D)$, notée $\left\langle\Delta_{1}, \Delta_{2}, \ldots, \Delta_{N}\right\rangle^{t}$, définie comme l'unique quotient irréductible de $\left\langle\Delta_{\sigma(1)}\right\rangle^{t} \times\left\langle\Delta_{\sigma(2)}\right\rangle^{t} \times \cdots \times\left\langle\Delta_{\sigma(N)}\right\rangle^{t}$, où $\sigma$ est une permutation de l'ensemble $\{1, \ldots, N\}$ telle que le multisegment $\Delta_{\sigma(1)}, \Delta_{\sigma(2)}, \ldots, \Delta_{\sigma(N)}$ soit rangé. La contragrédiente $\left\langle\Delta_{1}, \Delta_{2}, \ldots, \Delta_{N}\right\rangle^{t}$ est la représentation $\left\langle\widetilde{\Delta_{1}}, \widetilde{\Delta_{2}}, \ldots, \widetilde{\Delta_{N}}\right\rangle^{t}$.

La proposition suivante, dans le cas $D=F$ est montrée dans [21, 6.9.]. Dans le cas où $D \neq F$, la preuve est analogue et se trouve dans [12, 2.3.7].

Proposition 8.1. - Soit $\Delta_{1}, \Delta_{2}, \ldots, \Delta_{N}$ un multisegment rangé, avec $\Delta_{1}=\{b, b+$ $1, \ldots$, e $\}$ et $\Delta_{N}=\left\{b^{\prime}, b^{\prime}+1, \ldots, e^{\prime}\right\}$, alors

1. Si $\operatorname{Jac}_{\nu^{l}}\left(\left\langle\Delta_{1}, \Delta_{2}, \ldots, \Delta_{N}\right\rangle^{t}\right) \neq 0$, on a que $l \geq e^{\prime}$.

2. Si $\overline{\operatorname{Jac}}_{\nu^{l}}\left(\left\langle\Delta_{1}, \Delta_{2}, \ldots, \Delta_{N}\right\rangle^{t}\right) \neq 0$, on a que $l \leq b$.

\section{Fin de la preuve}

On s'est ramené aux cas où $\pi$ et $\pi^{\prime}$ sont des représentations très particulières, des représentations vérifiant les propriétés suivantes :

J.1 $\operatorname{Si~Jac}_{\chi}(\pi) \neq 0$ et $\chi$ cuspidale, alors $\chi \in\left\{\nu^{\frac{n+1}{2}}, \nu^{\frac{2 m-n+1}{2}}\right\}$,

J.2 si $\overline{\operatorname{Jac}}_{\chi}\left(\pi^{\prime}\right) \neq 0$ et $\chi$ cuspidale, alors $\chi \in\left\{\nu^{\frac{-m-1}{2}}, \nu^{\frac{m-2 n-1}{2}}\right\}$.

On va utiliser les propriétés de la section précédente pour terminer la preuve du théorème 6.1. On rappelle qu'on suppose $m>n$.

Soit $\pi \in \operatorname{Irr}\left(G_{n}\right)$ telle que $\operatorname{Jac}_{\nu \frac{n+1}{2}}(\pi) \neq 0$. Soit $a$ maximal tel que

$$
\pi \hookrightarrow \chi \times \chi \times \cdots \times \chi \times \rho,
$$

où on a fait le produit de $a$ fois le caractère $\chi=\nu^{\frac{n+1}{2}}$. On se trouve, avec les notations de la section 4, dans le cas A. On rappelle que l'on a deux possibilités, A1 et A2 :

CAs A.1. Il existe $\rho^{\prime} \in \operatorname{Irr}\left(G_{m-a}^{\prime}\right)$ avec

$$
\pi^{\prime} \hookrightarrow \rho^{\prime} \times \nu^{\frac{m-n}{2}} \chi^{-1} \times \cdots \times \nu^{\frac{m-n}{2}} \chi^{-1},
$$

où on a fait le produit de $a$ fois le caractère $\nu^{\frac{m-n}{2}} \chi^{-1}, a$ maximal et

$$
\operatorname{Hom}\left(\sigma_{n-a, m-a}, \nu^{\frac{-a}{2}} \rho \otimes \nu^{\frac{a}{2}} \rho^{\prime}\right) \neq 0
$$

(proposition 4.4).

Par hypothèse de récurrence, on a que :

$$
\pi^{\prime} \hookrightarrow \rho^{\prime} \times \nu^{\frac{m-2 n-1}{2}} \times \cdots \times \nu^{\frac{m-2 n-1}{2}}
$$

où

$$
\rho^{\prime}=\nu^{\frac{-a}{2}} \theta_{m-a}^{*}\left(\nu^{\frac{-a}{2}} \rho\right)=\left\langle\nu^{\frac{m-2 n-1}{2}}, \ldots, \nu^{\frac{-m+1}{2}}, \nu^{\frac{m-n}{2}} \widetilde{\Delta_{1}}, \ldots, \nu^{\frac{m-n}{2}} \widetilde{\Delta_{N}}\right\rangle^{t}
$$

si $\rho=\left\langle\Delta_{1}, \ldots, \Delta_{N}\right\rangle^{t}$, avec les notations de la section précédente.

Lemme 9.1. - Il n'existe pas de représentations irréductibles $\pi$ et $\pi^{\prime}$ satisfaisant aux conditions J.1, J.2 et (9.1).

$4^{\mathrm{e}}$ SÉRIE - TOME $41-2008-\mathrm{N}^{\circ} 5$ 
Démonstration. - D'après J.1, on sait que, si $\operatorname{Jac}_{\chi}(\pi) \neq 0$ et $\chi$ cuspidale, alors $\chi \in\left\{\nu^{\frac{n+1}{2}}, \nu^{\frac{2 m-n+1}{2}}\right\}$. Par 8.1.(1), tous les segments de $\rho$ finissent alors par $e_{i} \geq \frac{n+1}{2}$. Ainsi, par définition de $\rho^{\prime}$, tous les segments de $\rho^{\prime}$ commencent alors par $b_{i}^{\prime} \leq \frac{m-2 n-1}{2}$. De plus, puisque $m \neq n,\left\{\nu^{\frac{m-2 n-1}{2}}\right\}$ est un segment de $\rho^{\prime}$ et donc, par [13, Théorème 6.6.(3)], il existe $\tau^{\prime} \in \operatorname{Irr}\left(G_{m-a-1}^{\prime}\right)$ tel que $\rho^{\prime} \hookrightarrow \tau^{\prime} \times \nu^{\frac{m-2 n-1}{2}}$, ce qui contredit la maximalité de $a$.

Cas A.2. Alors on a bien (avec les notations de 4.4)

$$
\operatorname{Hom}\left({\overline{\tau^{\prime}}}_{b-a-1}, \nu^{\frac{m-n+a}{2}} \tilde{\chi} \times \cdots \times \nu^{\frac{m-n+a}{2}} \tilde{\chi} \otimes \nu^{\frac{a}{2}} \rho^{\prime}\right) \neq 0 .
$$

Dans ce cas, on a une proposition similaire à la proposition 4.4

Proposition 9.2. - Soient $\pi \in \operatorname{Irr}\left(G_{n}\right), \pi^{\prime} \in \operatorname{Irr}\left(G_{m}^{\prime}\right)$ telles que $\pi \otimes \pi^{\prime}$ soit un quotient de $\sigma_{n, m}$ satisfaisant aux conditions J.1 et J.2. Soit aussi $\chi=\nu^{\frac{n+1}{2}}$ un caractère de $G_{1}$. Alors $a=b-1$ où a et $b$ sont définis par les conditions suivantes :

1. Il existe $\rho \in \operatorname{Irr}\left(G_{n-a}\right)$ avec

$$
\pi \hookrightarrow \chi \times \chi \times \cdots \times \chi \times \rho
$$

où on a fait le produit de a fois le caractère $\chi$ et a est maximal.

2. Il existe $\rho^{\prime} \in \operatorname{Irr}\left(G_{m-b}^{\prime}\right)$ avec

$$
\pi^{\prime} \hookrightarrow \rho^{\prime} \times \nu^{\frac{m-n}{2}} \tilde{\chi} \times \cdots \times \nu^{\frac{m-n}{2}} \tilde{\chi}
$$

où on a fait le produit de b fois le caractère $\nu^{\frac{m-n}{2}} \tilde{\chi}$ et b est maximal.

De plus, on a

$$
\operatorname{Hom}\left(\sigma_{n-a, m-a-1}, \nu^{\frac{-a}{2}-1} \rho \otimes \nu^{\frac{a+1}{2}} \rho^{\prime}\right) \neq 0 .
$$

Démonstration. - On a que

$$
\begin{gathered}
\operatorname{Hom}\left(\operatorname{ind}_{P_{a, b-a}^{\prime}}^{G_{b}^{\prime}}\left(\nu^{\frac{m-n}{2}} \tilde{\chi} \times \cdots \times \nu^{\frac{m-n}{2}} \tilde{\chi} \otimes \nu^{\frac{a}{2}}{\overline{\tau^{\prime}}}_{b-a-1} \nu^{\frac{-a}{2}}\right),\right. \\
\left.\rho \otimes \nu^{\frac{m-n}{2}} \tilde{\chi} \times \cdots \times \nu^{\frac{m-n}{2}} \tilde{\chi} \otimes \rho^{\prime}\right) \neq 0,
\end{gathered}
$$

d'où, par définition de ${\overline{\tau^{\prime}}}_{b-a-1}$,

$$
\operatorname{Hom}\left(\operatorname{ind}_{P_{n-b-1, b-a-1}}^{G_{n-a}}\left(\nu^{\frac{b+1}{2}} \sigma_{n-b+1, m-b} \nu^{\prime \frac{-b}{2}} \otimes \nu^{\frac{-a}{2}} \chi \times \cdots \times \nu^{\frac{-a}{2}} \chi\right), \rho \otimes \rho^{\prime}\right) \neq 0,
$$

où on a fait le produit de $b-a-1$ fois le caractère $\nu^{\frac{-a}{2}} \chi$, et donc, par maximalité de $a$, on a $b=a+1$.

Ainsi, il existe $\rho^{\prime} \in \operatorname{Irr}\left(G_{m-a-1}^{\prime}\right)$ avec

$$
\pi^{\prime} \hookrightarrow \rho^{\prime} \times \nu^{\frac{m-n}{2}} \chi^{-1} \times \cdots \times \nu^{\frac{m-n}{2}} \chi^{-1},
$$

où on a fait le produit de $a+1$ fois le caractère $\nu^{\frac{m-n}{2}} \chi^{-1}, a$ maximal et

$$
\operatorname{Hom}\left(\sigma_{n-a, m-a-1}, \nu^{\frac{-a}{2}-1} \rho \otimes \nu^{\frac{a+1}{2}} \rho^{\prime}\right) \neq 0 .
$$

Alors, par hypothèse de récurrence, on a

$$
\operatorname{dim}\left(\operatorname{Hom}\left(\sigma_{n-a, m-a-1}, \nu^{\frac{-a}{2}-1} \rho \otimes \nu^{\frac{a+1}{2}} \rho^{\prime}\right)\right)=1,
$$


et

$$
\rho^{\prime}=\nu^{\frac{-a-1}{2}} \theta_{m-a}^{*}\left(\nu^{\frac{-a}{2}-1} \rho\right)=\left\langle\nu^{\frac{m-2 n-3}{2}}, \ldots, \nu^{\frac{-m+1}{2}}, \nu^{\frac{m-n}{2}} \widetilde{\Delta_{1}}, \ldots, \nu^{\frac{m-n}{2}} \widetilde{\Delta_{N}}\right\rangle^{t}
$$

si $\rho=\left\langle\Delta_{1}, \ldots, \Delta_{N}\right\rangle^{t}$.

Lemme 9.3. - Supposons que $\pi$ et $\pi^{\prime}$ sont deux représentations irréductibles qui satisfont aux conditions J.1, J.2 et telles que $\pi$ soit un sous-module de $\nu^{\frac{n+1}{2}} \times \cdots \times \nu^{\frac{n+1}{2}} \times \rho$, où on a fait le produit de a fois le caractère $\nu^{\frac{n+1}{2}}$, et $\pi^{\prime}$ soit un sous-module de $\rho^{\prime} \times \nu^{\frac{m-2 n-1}{2}} \times \cdots \times \nu^{\frac{m-2 n-1}{2}}$ où on a fait le produit de $a+1$ fois le caractère $\nu^{\frac{m-2 n-1}{2}} \chi^{-1}$ et

$$
\rho^{\prime}=\left\langle\nu^{\frac{m-2 n-3}{2}}, \ldots, \nu^{\frac{-m+1}{2}}, \nu^{\frac{m-n}{2}} \widetilde{\Delta_{1}}, \ldots, \nu^{\frac{m-n}{2}} \widetilde{\Delta_{N}}\right\rangle^{t}
$$

si $\rho=\left\langle\Delta_{1}, \ldots, \Delta_{N}\right\rangle^{t}$.

Alors $\pi^{\prime}=\theta_{m}^{*}(\pi)$.

Démonstration. - Notons $\pi_{1}^{\prime}$ l'unique sous-représentation irréductible de

$$
\rho^{\prime} \times \nu^{\frac{m-2 n-1}{2}} \times \cdots \times \nu^{\frac{m-2 n-1}{2}},
$$

où on a fait le produit de $a$ fois le caractère $\nu^{\frac{m-2 n-1}{2}}$, alors, d'après le corollaire 6.5 , on a que

$$
\begin{aligned}
\pi_{1}^{\prime} & =\nu^{\frac{-a}{2}} \theta_{m-a-1}^{*}\left(\nu^{\frac{-a}{2}} \pi\right) \\
& =\left\langle\nu^{\frac{m-2 n-3}{2}}, \ldots, \nu^{\frac{-m+1}{2}}, \nu^{\frac{m-n}{2}} \widetilde{\Delta_{1}^{\prime}}, \ldots, \nu^{\frac{m-n}{2}} \widetilde{\Delta_{N}^{\prime}}\right\rangle^{t}
\end{aligned}
$$

si $\pi=\left\langle\Delta_{1}^{\prime}, \ldots, \Delta_{N}^{\prime}\right\rangle^{t}$.

De plus, $\pi^{\prime}$ est l'unique sous-représentation irréductible de $\pi_{1}^{\prime} \times \nu^{\frac{m-2 n-1}{2}}$. Voyons finalement que $\pi^{\prime}=\theta_{m}^{*}(\pi)$.

Puisque d'après J.1, on sait que, si $\operatorname{Jac}_{\chi}(\pi) \neq 0$ et $\chi$ cuspidale, alors $\chi \in\left\{\nu^{\frac{n+1}{2}}, \nu^{\frac{2 m-n+1}{2}}\right\}$. Par la proposition 8.1(1), tous les segments de la forme $\Delta^{\prime}$ finissent alors par $e_{i} \geq \frac{n+1}{2}$. Ainsi, tous les segments de la forme $\nu^{\frac{m-n}{2}} \widetilde{\Delta_{i}^{\prime}}$ commencent par $b_{i}^{\prime} \leq \frac{m-2 n-1}{2}$ et, a fortiori, tous les segments de $\pi_{1}^{\prime}$ commencent par $b_{i}^{\prime} \leq \frac{m-2 n-1}{2}$.

Ainsi, par [13, Théorème 6.6.(2)], on a que

$$
\pi^{\prime}=\left\langle\nu^{\frac{m-2 n-1}{2}}, \nu^{\frac{m-2 n-3}{2}}, \ldots, \nu^{\frac{-m+1}{2}}, \nu^{\frac{m-n}{2}} \widetilde{\Delta_{1}}, \ldots, \nu^{\frac{m-n}{2}} \widetilde{\Delta_{N}}\right\rangle^{t}=\theta_{m}^{*}(\pi) .
$$

Il ne nous reste maintenant à traiter que les cas où $\pi$ et $\pi^{\prime}$ sont des représentations vérifiant les propriétés suivantes :

H.1 $\operatorname{Si~} \operatorname{Jac}_{\chi}(\pi) \neq 0$ et $\chi$ cuspidale, alors $\chi=\nu^{\frac{2 m-n+1}{2}}$,

H.2 si $\overline{\operatorname{Jac}}_{\chi}\left(\pi^{\prime}\right) \neq 0$ et $\chi$ cuspidale, alors $\chi=\nu^{\frac{-m-1}{2}}$.

Proposition 9.4. - Il n'existe pas de représentations irréductibles $\pi \in \operatorname{Irr}\left(G_{n}\right)$ et $\pi^{\prime} \in$ $\operatorname{Irr}\left(G_{m}^{\prime}\right), m>n$, satisfaisant aux conditions $H .1$ et $H .2$ et telles que $\operatorname{Hom}\left(\sigma_{n}, \pi \otimes \pi^{\prime}\right) \neq 0$. 
Soit $\pi \in \operatorname{Irr}\left(G_{n}\right)$ vérifiant H.1 et soit $a$ maximal tel que

$$
\pi \hookrightarrow \chi \times \chi \times \cdots \times \chi \times \rho,
$$

où on a fait le produit de $a$ fois le caractère $\chi=\nu^{\frac{2 m-n+1}{2}}$. On a alors, avec les notations de la section 4 , que

Cas A. Ou bien, $\operatorname{Hom}\left(\tau_{a}, \chi \times \chi \times \cdots \times \chi \otimes \rho \otimes \pi^{\prime}\right) \neq 0$.

CAs B. Ou bien, Hom $\left(\tau_{a-1}, \chi \times \chi \times \cdots \times \chi \otimes \rho \otimes \pi^{\prime}\right) \neq 0$.

C'est-à-dire :

Cas A. Puisque $m \neq n$, on est bien dans le cas A.1 et donc, d'après 4.4, il existe $\rho^{\prime} \in$ $\operatorname{Irr}\left(G_{m-a}^{\prime}\right)$ avec

$$
\pi^{\prime} \hookrightarrow \rho^{\prime} \times \nu^{\frac{m-n}{2}} \chi^{-1} \times \cdots \times \nu^{\frac{m-n}{2}} \chi^{-1},
$$

où on a fait le produit de $a$ fois le caractère $\nu^{\frac{m-n}{2}} \chi^{-1}, a$ maximal et

$$
\operatorname{Hom}\left(\sigma_{n-a, m-a}, \nu^{\frac{-a}{2}} \rho \otimes \nu^{\frac{a}{2}} \rho^{\prime}\right) \neq 0 .
$$

Ceci n'est pas possible. En effet, par récurrence, on a que :

$$
\pi^{\prime} \hookrightarrow \rho^{\prime} \times \nu^{\frac{-m-1}{2}} \times \cdots \times \nu^{\frac{-m-1}{2}}
$$

et

$$
\rho^{\prime}=\left\langle\nu^{\frac{m-2 n-1}{2}}, \ldots, \nu^{\frac{-m+1}{2}}, \nu^{\frac{m-n}{2}} \widetilde{\Delta_{1}}, \ldots, \nu^{\frac{m-n}{2}} \widetilde{\Delta_{N}}\right\rangle^{t}
$$

si $\rho=\left\langle\Delta_{1}, \ldots, \Delta_{N}\right\rangle^{t}$.

Il y a ainsi des segments de $\pi^{\prime}$ commençant par $x_{i}>\frac{-m-1}{2}$ ce qui, par la proposition 8.1(2), contredit H.2.

CAs B. Sinon, montrons qu'il existe $\rho^{\prime} \in \operatorname{Irr}\left(G_{m-a+1}^{\prime}\right)$ avec

$$
\pi^{\prime} \hookrightarrow \rho^{\prime} \times \nu^{\frac{m-n}{2}} \chi^{-1} \times \cdots \times \nu^{\frac{m-n}{2}} \chi^{-1},
$$

où on a fait le produit de $a-1$ fois le caractère $\nu^{\frac{m-n}{2}} \chi^{-1}$, a maximal et

$$
\operatorname{Hom}\left(\sigma_{n-a, m-a+1}, \nu^{\frac{-a}{2}} \rho \otimes \nu^{\frac{a+1}{2}} \rho^{\prime}\right) \neq 0 .
$$

En effet, $\operatorname{Hom}\left(\tau_{a-1}, \chi \times \chi \times \cdots \times \chi \otimes \rho \otimes \pi^{\prime}\right) \neq 0$ implique que

$$
\begin{aligned}
\operatorname{Hom}\left(\operatorname{ind}_{P_{a-1, m-a+1}^{\prime}}^{G_{m}^{\prime}}\right. & \left(\nu^{\frac{m-n}{2}} \chi^{-1} \times \cdots \times \nu^{\frac{m-n}{2}} \chi^{-1} \otimes\right. \\
& \left.\left.\nu^{\frac{a}{2}} \sigma_{n-a, m-a+1} \nu^{\prime \frac{-a-1}{2}}\right), \rho \otimes \pi^{\prime}\right) \neq 0 .
\end{aligned}
$$

Soit $b$ maintenant maximal tel qu'il existe une représentation irréductible $\rho^{\prime}$ de $G_{m-b}^{\prime}$ avec $\pi^{\prime}$ quotient de

$$
\nu^{\frac{m-n}{2}} \chi^{-1} \times \cdots \times \nu^{\frac{m-n}{2}} \chi^{-1} \times \rho^{\prime}
$$

où on a fait le produit de $b$ fois le caractère $\nu^{\frac{m-n}{2}} \chi^{-1}$. D'après le corollaire 1.2, on a une flèche non nulle

$$
\bar{r}_{b, m-b}^{G_{m}^{\prime}}\left(\pi^{\prime}\right) \hookrightarrow \nu^{\frac{m-n}{2}} \chi^{-1} \times \cdots \times \nu^{\frac{m-n}{2}} \chi^{-1} \otimes \rho^{\prime} .
$$

Puisque $\chi \neq \nu^{\frac{n+1}{2}}$, on montre, comme dans 4.4, que $b=a-1$ et donc

$$
\operatorname{Hom}\left(\sigma_{n-a, m-a+1}, \nu^{\frac{-a}{2}} \rho \otimes \nu^{\frac{a+1}{2}} \rho^{\prime}\right) \neq 0 \text {. }
$$


Ainsi, il existe $\rho^{\prime} \in \operatorname{Irr}\left(G_{m-a+1}^{\prime}\right)$ avec

$$
\pi^{\prime} \hookrightarrow \rho^{\prime} \times \nu^{\frac{m-n}{2}} \chi^{-1} \times \cdots \times \nu^{\frac{m-n}{2}} \chi^{-1},
$$

où on a fait le produit de $a-1$ fois le caractère $\nu^{\frac{m-n}{2}} \chi^{-1}, a$ maximal et

$$
\operatorname{Hom}\left(\sigma_{n-a, m-a+1}, \nu^{\frac{-a}{2}} \rho \otimes \nu^{\frac{a+1}{2}} \rho^{\prime}\right) \neq 0 .
$$

Alors, par hypothèse de récurrence, on a

$$
\pi^{\prime} \hookrightarrow \rho^{\prime} \times \nu^{\frac{-m-1}{2}} \times \cdots \times \nu^{\frac{-m-1}{2}},
$$

et

$$
\rho^{\prime}=\left\langle\nu^{\frac{m-2 n-1}{2}}, \ldots, \nu^{\frac{-m-1}{2}}, \nu^{\frac{m-n}{2}} \widetilde{\Delta_{1}}, \ldots, \nu^{\frac{m-n}{2}} \widetilde{\Delta_{N}}\right\rangle^{t}
$$

si $\rho=\left\langle\Delta_{1}, \ldots, \Delta_{N}\right\rangle^{t}$. Si $n \neq m$, on trouve ainsi des segments de $\pi^{\prime}$ commençant par $x_{i}>\frac{-m-1}{2}$ ce qui, à nouveau par 8.1(2), contredit H.2.

\section{RÉFÉRENCES}

[1] I. N. BER NŠTEǏN, Representations of $p$-adic groups, Notes by K.E. Rumelhart, Harvard Univ., 1992.

[2] I. N. BernšteǏn, A. V. Zelevinskir̆, Representations of the group GL $(n, F)$, where $F$ is a local non-Archimedean field, Uspehi Mat. Nauk 31 (1976), 5-70.

[3] N. Bourbaki, Algèbre, Chapitre 2, Hermann, Paris, 1970.

[4] C. J. Bushnell, Representations of reductive $p$-adic groups : localization of Hecke algebras and applications, J. London Math. Soc. 63 (2001), 364-386.

[5] W. Casselman, Introduction to the theory of admissible representations of $p$-adic reductive groups, prépublication http://www.math.ubc.ca/ ${ }^{\sim} \mathrm{cass} / \mathrm{research}$. html.

[6] R. Godement, H. Jacquet, Zeta functions of simple algebras, Lecture Notes in Math., Vol. 260, Springer, 1972.

[7] M. Harris, S. S. Kudla, W. J. Sweet, Theta dichotomy for unitary groups, J. Amer. Math. Soc. 9 (1996), 941-1004.

[8] M. HAR RIS, R. TAYLOR, The geometry and cohomology of some simple Shimura varieties, Annals of Mathematics Studies 151, Princeton University Press, 2001.

[9] G. Henniart, Une preuve simple des conjectures de Langlands pour GL $(n)$ sur un corps $p$-adique, Invent. Math. 139 (2000), 439-455.

[10] S. S. Kudla, Notes on the local theta correspondence, in Lectures notes from the European School on Group Theory, Schloß Hirschberg, Germany, 1966.

[11] S. S. KudLA, On the local theta-correspondence, Invent. Math. 83 (1986), 229-255.

[12] A. Mínguez, Correspondance de Howe $l$-modulaire : paires duales de type II, Thèse, Université d'Orsay, 2006.

[13] A. Mínguez, Sur l'irréductibilité d'une induite parabolique, prépublication à paraître dans $J$. reine angew. Math. .

$4^{\mathrm{e}}$ SÉRIE - TOME $41-2008-\mathrm{N}^{\circ} 5$ 
[14] C. Mqeglin, M.-F. Vignéras, J.-L. Waldspurger, Correspondances de Howe sur un corps p-adique, Lecture Notes in Math. 1291, Springer, 1987.

[15] G. Muić, Howe correspondence for discrete series representations; the case of $(\mathrm{Sp}(n), \mathrm{O}(V)), J$ reine angew. Math. 567 (2004), 99-150.

[16] G. Muić, Theta lifts of tempered representations for dual pairs $(S p(2 n), O(V))$, à paraître dans Canad. J. Math. .

[17] A. J. Silberger, The Langlands quotient theorem for $p$-adic groups, Math. Ann. 236 (1978), 95-104.

[18] M. TADIĆ, Induced representations of $\operatorname{GL}(n, A)$ for $p$-adic division algebras, J. reine angew. Math. 405 (1990), 48-77.

[19] J.-L. Waldspurger, Démonstration d'une conjecture de dualité de Howe dans le cas $p$-adique, $p \neq 2$, in Festschrift in honor of I. I. Piatetski-Shapiro on the occasion of his sixtieth birthday, Israel Math. Conf. Proc. 2, Weizmann, 1990, 267-324.

[20] T. Watanabe, The local theta correspondence of irreducible type 2 dual reductive pairs, Tohoku Math. J. 47 (1995), 521-540.

[21] A. V. Zelevinsky, Induced representations of reductive $p$-adic groups. II. On irreducible representations of GL(n), Ann. Sci. École Norm. Sup. 13 (1980), 165-210.

(Manuscrit reçu le 21 août 2007 ;

accepté, après révision, le 25 mars 2008.)

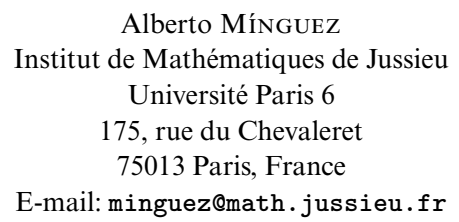

